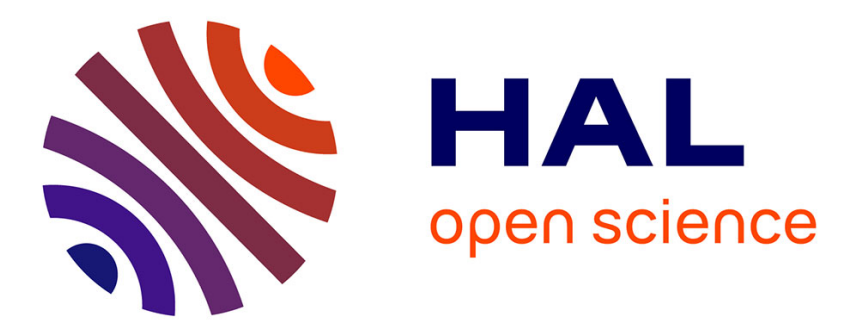

\title{
Computational stochastic homogenization of heterogeneous media from an elasticity random field having an uncertain spectral measure Christian Soize
}

\section{- To cite this version: \\ Christian Soize. Computational stochastic homogenization of heterogeneous media from an elasticity random field having an uncertain spectral measure. Computational Mechanics, 2021, 68, pp.1003-1021. 10.1007/s00466-021-02056-8 . hal-03321743}

\section{HAL Id: hal-03321743 \\ https://hal.science/hal-03321743}

Submitted on 18 Aug 2021

HAL is a multi-disciplinary open access archive for the deposit and dissemination of scientific research documents, whether they are published or not. The documents may come from teaching and research institutions in France or abroad, or from public or private research centers.
L'archive ouverte pluridisciplinaire $\mathbf{H A L}$, est destinée au dépôt et à la diffusion de documents scientifiques de niveau recherche, publiés ou non, émanant des établissements d'enseignement et de recherche français ou étrangers, des laboratoires publics ou privés. 


\title{
Computational stochastic homogenization of heterogeneous media from an elasticity random field having an uncertain spectral measure
}

\author{
Christian Soize ${ }^{*, a}$ \\ ${ }^{a}$ Univ Gustave Eiffel, MSME UMR 8208, 5 Bd Descartes, 77454 Marne-La-Vallée, France
}

\begin{abstract}
This paper presents the computational stochastic homogenization of a heterogeneous 3D-linear anisotropic elastic microstructure that cannot be described in terms of constituents at microscale, as live tissues. The random apparent elasticity field at mesoscale is then modeled in a class of non-Gaussian positive-definite tensor-valued homogeneous random fields. We present an extension of previous works consisting of a novel probabilistic model to take into account uncertainties in the spectral measure of the random apparent elasticity field. A probabilistic analysis of the random effective elasticity tensor at macroscale is performed as a function of the level of spectrum uncertainties, which allows for studying the scale separation and the representative volume element size in a robust probabilistic framework.
\end{abstract}

Key words: Stochastic homogenization, Non-Gaussian random fields, Uncertain spectral measure, Heterogeneous microstructure, Uncertainty Quantification, Live tissues

\section{Introduction}

The homogenization of linear elastic materials with heterogeneous microstructures composed of several phases with well defined constituents (from a continuum mechanics point of view) and the calculation of the macroscopic properties (effective properties) have received considerable attention (see for instance $[1,2,3,4,5,6,7,8,9,10,11]$ ), for stochastic homogenization (see $[12,13,14,15,16,17,18,19])$, for computational multiresolution materials and multiscale method (see for instance [20, 21, 22, 23, 24, 25, 26]), and more recently, for data-driven and machine learning approaches applied to heterogeneous materials (see for instance $[27,28,29$, 30, 31, 32, 33]).

In linear elasticity, the random microstructure is considered as homogenizable if there exists a Representative Volume Element (RVE) for which the random fluctuations of the random effective stiffness tensor around its statistical mean value are "negligible". The analysis of the RVE size has received a particular attention (see for instance [34, 35, 36, 37, 38, 39]). Often, the statisticsbased bounding techniques only use the lower-order statistics (first- and second-order moments) and the probability distributions, which give the detailed probabilistic information, are not taken into account.

${ }^{*}$ Corresponding author: Christian Soize, christian.soize@univ-eiffel.fr Preprint of the published paper in Computational Machanics, 2021. 


\begin{tabular}{|c|c|c|}
\hline Scale & SIMPLE MICROSTRUCTURE & COMPLEX MICROSTRUCTURE \\
\hline$\underset{\text { Macroscale }}{\mathbf{L}}$ & Effective properties & Effective properties \\
\hline$\ell_{\text {RVE size }}$ & \multirow{2}{*}{$\begin{array}{l}\text { Use of a method } \\
\text { for scale change }\end{array}$} & $\uparrow \begin{array}{l}\text { Use of a method } \\
\text { for scale change }\end{array}$ \\
\hline$\lambda_{\text {Correlation length }}^{\lambda}$ & & $\begin{array}{l}\text { Stochastic model of the apparent } \\
\text { properties at mesoscale }\end{array}$ \\
\hline $\begin{array}{l}\text { d } \\
\text { Size of } \\
\text { heterogeneous } \\
\text { details }\end{array}$ & $\begin{array}{l}\text { Stochastic model deduced from the } \\
\text { stochastic model of the geometry and } \\
\text { of the constituents of the microstructure }\end{array}$ & $\begin{array}{l}\text { Stochastic model cannot be constructed } \\
\text { in terms of the constituents due to the } \\
\text { complexity of the microstructure }\end{array}$ \\
\hline $\begin{array}{c}\quad \mathrm{d}_{0} \\
\text { Lower bound }\end{array}$ & \multirow{2}{*}{$\begin{array}{l}\text { No significant statistical coupling } \\
\text { with the inferior scale }\end{array}$} & \multirow{2}{*}{$\begin{array}{l}\text { Significant statistical coupling } \\
\text { with inferior scales }\end{array}$} \\
\hline$\mu$ & & \\
\hline
\end{tabular}

Figure 1: Typical scales of the domain for the boundary value problem; types of stochastic modeling for a simple microstructure and for a complex microstructure; notion of apparent and effective properties. Figure from [48].

For linear elastic heterogeneous microstructure, which cannot be described in terms of constituents at microscale (such as living tissues for which the constituents/phases cannot be described at microscale), a stochastic homogenization has been proposed in [40]. A probabilistic analysis of the RVE and of the random effective elasticity tensor has been carried out by using a prior probabilistic model of the random apparent elasticity field at mesoscale. In such a case, the hyperparameters of such a prior probabilistic model can be identified from experiments by solving statistical inverse problems as proposed in [32, 33, 41, 42, 43, 44, 45, 46, 47]. Fig. 1 shows the typical scales of the domain for the boundary value problems in the framework of multiscale modeling of heterogeneous materials. It can be seen the types of stochastic modeling for a simple microstructure and for a complex microstructure, and the notion of apparent and effective properties. In particular, it can be seen that, for a heterogeneous complex microstructure, a stochastic model of the apparent properties of the microstructure can be constructed at the mesoscale that corresponds to the scale of the spatial correlation length of the microstructure. This case constitutes the framework of the present paper.

The construction of prior probabilistic models requires the use of the random fields theory that has extensively been developed $[49,50,51,52,53]$, in particular in the context of continuum physics $[18,48,54,55]$. However, such a construction of the random apparent tensor-valued elasticity field at mesoscale requires specific developments due to the symmetry and positiveness properties of this random field that is non-Gaussian. Such a construction was proposed in [56] for elliptic stochastic partial differential operators and was used for performing the probabilistic analysis of stochastic homogenization and the RVE in [40]. This type of construction has been extended $[48,55,57,58,59]$ in order to take into account more complex situations for which there is a material symmetry (see for instance [60] for the characterization of material symmetries), which belongs to a given symmetry class for the mean value of the random elasticity field and considering the statistical fluctuations either in the same symmetry class, or in another sym- 
metry class, or in a mixture of two symmetry classes.

Objective and novelties of the paper. The objective of this paper is the computational stochastic homogenization of a 3D-linear elastic heterogeneous microstructure. The apparent elasticity field at mesoscale is modeled by a non-Gaussian positive-definite fourth-order tensor-valued homogeneous random field. This paper present an extension of the works [47, 48, 56, 57, 61] devoted to random field representations for stochastic elliptic boundary value problems and computational stochastic homogenization. We propose a novel probabilistic modeling to take into account uncertainties in the spectral measure of the random apparent elasticity field and we solve a stochastic elliptic boundary value problem (BVP) to perform the computational stochastic homogenization. Mathematical aspects associated with this paper can be found in [62].

The computational stochastic homogenization with uncertain spectral measure we propose allows several problems to be analyzed and solved in the context of the given hypotheses on the microstructures.

- First of all, the approach allows a robust probabilistic analysis of the random effective elasticity tensor to be carried out with respect to uncertainties of the spectral measure of the random apparent elasticity field. This spectral measure drives the spatial correlation structure in terms of spatial correlation lengths and the spectral distribution of the statistical fluctuations at mesoscale. - Then, the framework used also allows a robust probabilistic analysis of the RVE size to be performed and therefore, to study the scale separation.

- Finally, an important aspect is related to the robust experimental identification of the hyperparameters of the prior probability model of the random apparent elasticity field at mesoscale from multiscale measurements (macroscale and micro/mesoscale). Such a case is particularly important when the experimental test specimen is smaller that the RVE. In that condition, at macroscale, the effective elasticity tensor has significant statistical fluctuations. So it is very important to be able to identify experimentally and in a robust way the prior probability model of the random apparent elasticity field at mesoscale in order to be able to use the identified random field to make the computational stochastic homogenization on a RVE for which there will be a scale separation.

Organization of the paper. In Section 1, we present the principle of the construction of the random apparent elasticity field $\left\{\widetilde{\mathbb{C}}(\mathbf{x}), \mathbf{x} \in \mathbb{R}^{3}\right\}$ at mesoscale, in presence of uncertainties in its spectral measure. Section 3 deals with the definition of the stochastic elliptic boundary value problem at mesoscale, which is used for performing the computational stochastic homogenization and for constructing the random effective elasticity tensor $\widetilde{\mathbb{C}}^{\text {eff }}$ at macroscale. Section 4 is devoted to the construction of the random apparent elasticity field with an uncertain spectral measure. In Section 5, we analyze the solution of the stochastic boundary value problem and we introduce the random eigenvalues of the random effective elasticity matrix. In Section 6, we define the models and data for performing the computational stochastic homogenization with uncertain spectral measure. The estimation of the parameters of the computational stochastic homogenization is performed in Section 7 on the basis of a convergence analysis. Finally, the numerical results with a discussion of the computational stochastic homogenization are presented in Section 8. 


\section{Notations}

The following notations are used:

$x$ : lower-case Latin or Greek letters are deterministic real variables.

$\mathbf{x}$ : boldface lower-case Latin, Greek, and calligraphic letters are deterministic vectors.

$X$ : upper-case Latin, Greek, and calligraphic letters are realvalued random variables.

$\mathbf{X}$ : boldface upper-case Latin or Greek letters are vectorvalued random variables.

$[x]$ : lower-case Latin of Greek letters between brackets are deterministic matrices.

$[\mathbf{X}]$ : boldface upper-case letters between brackets are matrixvalued random variables.

[y]: deterministic matrix-valued parameter that controls the spectral measure uncertainties.

[Y]: random matrix modeling $[y]$.

$\mathbb{C}$ : fourth-order tensor-valued random field.

$\mathbb{C}(\cdot ;[y])$ : parameterized random apparent elasticity field.

$\mathbf{C}(\cdot ;[y])$ : normalization of random field $\mathbb{C}(\cdot ;[y])$.

$\widetilde{\mathbb{C}}$ : random apparent elasticity field equal to $\mathbb{C}(\cdot ;[\mathbf{Y}])$.

$\mathbb{C}^{\text {eff }}([y])$ : parameterized random effective elasticity tensor.

$\widetilde{\mathbb{C}}^{\text {eff }}$ : random effective elasticity tensor equal to $\mathbb{C}^{\text {eff }}([\mathbf{Y}])$.

$\mathbb{N}, \mathbb{R}$ : set of all the integers, set of all the real numbers.

$\mathbb{R}^{n}$ : Euclidean vector space on $\mathbb{R}$ of dimension $n$.

$\mathbb{M}_{n, m}$ : set of all the $(n \times m)$ real matrices.

$\mathbb{M}_{n}$ : set of all the square $(n \times n)$ real matrices.

$\mathbb{M}_{n}^{S}$ : set of all the symmetric $(n \times n)$ real matrices.

$\mathbb{M}_{n}^{+}$: set of all the positive-definite $(n \times n)$ real matrices.

$\left[I_{n}\right]$ : identity matrix in $\mathbb{M}_{n}$.

$\mathbf{x}=\left(x_{1}, \ldots, x_{n}\right)$ : point in $\mathbb{R}^{n}$.

$\left\langle\mathbf{x}, \mathbf{x}^{\prime}\right\rangle_{2}=x_{1} x_{1}^{\prime}+\ldots+x_{n} x_{n}^{\prime}$ : inner product in $\mathbb{R}^{n}$.

$\|\mathbf{x}\|_{2}$ : norm in $\mathbb{R}^{n}$ such that $\|\mathbf{x}\|^{2}=\langle\mathbf{x}, \mathbf{x}\rangle_{2}$.

$\|[a]\|_{2}=\sup _{\mathbf{x}}\left\{\|[a] \mathbf{x}\|_{2} /\|\mathbf{x}\|_{2}\right\}$ for $[a] \in \mathbb{M}_{n}$ and $\mathbf{x} \in \mathbb{R}^{n}$.

$[x]^{T}$ : transpose of matrix $[x]$.

$\operatorname{tr}\{[x]\}$ : trace of the square matrix $[x]$.

$\left\langle[x],\left[x^{\prime}\right]\right\rangle_{F}=\operatorname{tr}\left\{[x]^{T}\left[x^{\prime}\right]\right\}$, inner product in $\mathbb{M}_{n, m}$.

$\|[x]\|_{F}$ : Frobenius norm such that $\|[x]\|_{F}^{2}=\langle[x],[x]\rangle_{F}$.

$\mathbb{1}_{B}$ : indicatrix function of set $B$.

$\iota$ : imaginary unit.

$\delta$ : dispersion parameter such that $\delta=\delta_{1}=\delta_{2}=\delta_{3}$, which controls the level of the spectral measure uncertainties.

$\delta_{j}$ : dispersion parameter controlling the uncertainties in the spectral measure for space coordinate $j$.

$\delta_{c}$ : dispersion parameter controlling the statistical fluctuations of the random apparent elasticity field.

$\delta_{k k^{\prime}}$ : Kronecker's symbol. 
$\delta_{\mathbf{x}_{0}}:$ Dirac measure at point $\mathbf{x}_{0}$.

a.s: almost surely.

$E$ : mathematical expectation.

$(\Theta, \mathcal{T}, \mathcal{P})$ : probability space.

\section{Principle of the construction}

The physical space $\mathbb{R}^{3}$ is referred to a Cartesian reference system for which the generic point is $\mathbf{x}=\left(x_{1}, x_{2}, x_{3}\right)$. At mesoscale the linear elastic heterogeneous medium is described by the random apparent elasticity field, $\left\{\widetilde{\mathbb{C}}(\mathbf{x}), \mathbf{x} \in \mathbb{R}^{3}\right\}$, which is a non-Gaussian fourth-order tensorvalued random field $\widetilde{\mathbb{C}}=\left\{\widetilde{\mathbb{C}}_{i j p q}\right\}_{i j p q}$ with $i, j, p$, and $q$ in $\{1,2,3\}$.

As explained in Section 1, we could consider a given symmetry class for the mean value of the random elasticity field and consider the statistical fluctuations either in the same symmetry class, or in another symmetry class, or in a mixture of two symmetry classes (as proposed in $[57,58,48])$. However, although the case of the mixture of two symmetry classes does not pose any theoretical problems and could very well have been considered to implement the proposed model for the uncertain spectral measure, this situation would have considerably complicated the presentation of the proposed construction to the detriment of readability. The extension of the developments presented can therefore be made without particular theoretical difficulties and we consider the following more simple case. We start the construction of this random field with the formulation proposed in [56]. It is assumed that the mean value of random apparent elasticity field $\widetilde{\mathbb{C}}$ is independent of $\mathbf{x}$ and belongs to any material symmetry class (isotropic, transverse isotropic, orthotropic, etc.). The statistical fluctuations of $\widetilde{\mathbb{C}}$ around the mean value are assumed to be anisotropic and statistically homogeneous in $\mathbb{R}^{3}$. This means that the mean elasticity field $E\{\widetilde{\mathbb{C}}\}$ is constant and can be chosen in any symmetry class (in the application presented in Section 6, we will use the isotropic class), and that the stochastic elasticity field $\widetilde{\mathbb{C}}-E\{\widetilde{\mathbb{C}}\}$ is anisotropic almost-surely and is statistically homogeneous (in the strong sense for the shift group on $\mathbb{R}^{3}$ defined by the shifts $\mathbf{x} \mapsto \mathbf{x}+\zeta$, for all $\zeta$ in $\mathbb{R}^{3}$ ).

An important quantity that controls the mesoscale statistical fluctuations is the spectral measure of $\widetilde{\mathbb{C}}$, which allows the spatial correlation structure to be described and that we will assumed to be uncertain in this paper.

We introduce a parameterization of the spectral measure of $\widetilde{\mathbb{C}}$, which involves a matrix-valued parameter $[y]$ belonging to an admissible set $C_{y}$. The uncertain spectral measure is obtained by modeling $[y]$ by a matrix-valued random variable $[\mathbf{Y}]$ whose support of its probability distribution is $C_{y}$. We then construct a non-Gaussian positive-definite fourth-order tensor-valued homogeneous random field $\mathbb{C}(\cdot ;[y])$ that is parameterized with $[y]$ such that $\widetilde{\mathbb{C}}=\mathbb{C}(\cdot ;[\mathbf{Y}])$.

For all $\mathbf{x}$ fixed in $\mathbb{R}^{3}$ and $[y]$ fixed in $C_{y}$, the fourth-order random tensor $\mathbb{C}(\mathbf{x} ;[y])$ verifies the usual symmetry and positiveness properties. The representation in Voigt notation is used for the constitutive equation. Let $\mathbf{i}=(i, j)$ with $1 \leq i \leq j \leq 3$ and $\mathbf{j}=(p, q)$ with $1 \leq p \leq q \leq 3$ be the indices with values in $\{1, \ldots, 6\}$, which allow for defining the $\mathbb{M}_{6}^{+}$-valued random matrix $[\mathbb{C}(\mathbf{x} ;[y])]$ such that $[\mathbb{C}(\mathbf{x} ;[y])]_{\mathbf{i j}}=\mathbb{C}_{i j p q}(\mathbf{x} ;[y])$.

For fixed $[y]$ in $C_{y}$, the random effective elasticity tensor $\mathbb{C}^{\text {eff }}([y])$ satisfies the symmetry and positive-definiteness properties [63]. We can thus define the $\mathbb{M}_{6}^{+}$-valued random effective elasticity matrix $\left[\mathbb{C}^{\text {eff }}([y])\right]$ associated with random tensor $\mathbb{C}^{\text {eff }}([y])$, which is such that $\left[\mathbb{C}^{\text {eff }}([y])\right]_{\mathrm{ij}}=$ $\mathbb{C}_{i j \ell r}^{\text {eff }}([y])$ in which $\mathbf{i}=(i, j)$ with $1 \leq i \leq j \leq 3$ and $\mathbf{j}=(\ell, r)$ with $1 \leq \ell \leq r \leq 3$. The parameterized effective elasticity matrix $\left[\mathbb{C}^{\text {eff }}([y])\right]$ is a random matrix in $\mathbb{M}_{6}^{+}$, which is obtained by 
stochastic homogenization solving a stochastic elliptic BVP on a bounded domain $\Omega$ of $\mathbb{R}^{3}$. The random effective elasticity matrix $\left[\widetilde{\mathbb{C}}^{\text {eff }}\right]$, corresponding to the random apparent elasticity field with uncertain spectral measure, is then given by $\left[\widetilde{\mathbb{C}}^{\text {eff }}\right]=\left[\mathbb{C}^{\text {eff }}([\mathbf{Y}])\right]$.

\section{Stochastic elliptic boundary value problem for the computational stochastic homoge- nization and random effective elasticity tensor}

The heterogeneous linear elastic microstructure occupies the 3-D bounded open domain $\Omega \subset \mathbb{R}^{3}$ with boundary $\partial \Omega$. The homogenization method on $\Omega$ is that proposed in [63] for homogeneous deformations on the boundary $\partial \Omega$ (and that we have already used in [40]). The convention for summations over repeated Latin indices $j, p$, and $q$ taking values in $\{1,2,3\}$ is used. Let $[y]$ be fixed in $C_{y}$. For all $\ell$ and $r$ in $\{1,2,3\}$, we have to find the $\mathbb{R}^{3}$-valued random field $\left\{\widetilde{\mathbf{U}}^{\ell r}(\mathbf{x})=\left(\widetilde{U}_{1}^{\ell r}(\mathbf{x}), \widetilde{U}_{2}^{\ell r}(\mathbf{x}), \widetilde{U}_{3}^{\ell r}(\mathbf{x})\right), \mathbf{x} \in \bar{\Omega}\right\}$, defined on a probability space $(\Theta, \mathcal{T}, \mathcal{P})$, indexed by $\bar{\Omega}=\Omega \cup \partial \Omega$, such that for $i \in\{1,2,3\}$ and almost surely,

$$
\begin{aligned}
&-\frac{\partial}{\partial x_{j}}\left(\mathbb{C}_{i j p q}(\mathbf{x} ;[y]) \varepsilon_{p q}\left(\widetilde{\mathbf{U}}^{\ell r}(\mathbf{x})\right)=0\right., \quad \forall \mathbf{x} \in \Omega, \\
& \widetilde{\mathbf{U}}^{\ell r}(\mathbf{x})=\widetilde{\mathbf{u}}_{0}^{\ell r}(\mathbf{x}) \quad, \quad \forall \mathbf{x} \in \partial \Omega,
\end{aligned}
$$

in which the strain tensor is written as $\varepsilon_{p q}(\mathbf{u})=\left(\partial u_{p} / \partial x_{q}+\partial u_{q} / \partial x_{p}\right) / 2$ for $\mathbf{u}=\left(u_{1}, u_{2}, u_{3}\right)$ and where for all $\mathbf{x}$ in $\partial \Omega, \widetilde{\mathbf{u}}_{0}^{\ell r}(\mathbf{x})=\left(\widetilde{u}_{0,1}^{\ell r}(\mathbf{x}), \widetilde{u}_{0,2}^{\ell r}(\mathbf{x}), \widetilde{u}_{0,3}^{\ell r}(\mathbf{x})\right)$ is defined by

$$
\widetilde{u}_{0, j}^{\ell r}(\mathbf{x})=\left(\delta_{j \ell} x_{r}+\delta_{j r} x_{\ell}\right) / 2 \quad, \quad j \in\{1,2,3\},
$$

with $\delta_{j \ell}$ the Kronecker symbol. For $[y]$ fixed in $C_{y}$, for $i, j, \ell$, and $r$ in $\{1,2,3\}$ the component $\mathbb{C}_{i j \ell r}^{\text {eff }}([y])$ of the random fourth-order effective elasticity tensor $\mathbb{C}^{\text {eff }}([y])$ is defined by

$$
\mathbb{C}_{i j \ell r}^{\mathrm{eff}}([y])=\frac{1}{|\Omega|} \int_{\Omega} \mathbb{C}_{i j p q}(\mathbf{x} ;[y]) \varepsilon_{p q}\left(\widetilde{\mathbf{U}}^{\ell r}(\mathbf{x})\right) d \mathbf{x}
$$

in which $\widetilde{\mathbf{U}}^{\ell r}$ is the $\mathbb{R}^{3}$-valued random field that satisfies Eqs. (1) to (3) and where $|\Omega|=\int_{\Omega} d \mathbf{x}$. The fourth-order effective tensor $\mathbb{C}^{\text {eff }}([y])$ satisfies the symmetry and positive-definiteness properties. We can thus define the $\mathbb{M}_{6}^{+}$-valued random effective elasticity matrix $\left[\mathbb{C}^{\text {eff }}([y])\right]$ that is associated with random tensor $\mathbb{C}^{\text {eff }}([y])$ as explained in Section 2.

\section{Construction of random apparent elasticity field with uncertain spectral measure}

\subsection{Normalization of random field $[\mathbb{C}(\cdot ;[y])]$}

Let $[\underline{\mathbb{C}}]$ be a given matrix in $\mathbb{M}_{6}^{+}$independent of $\mathbf{x}$ and $[y]$. We define $\left\{[\mathbb{C}(\mathbf{x} ;[y])], \mathbf{x} \in \mathbb{R}^{3}\right\}$ as a non-Gaussian $\mathbb{M}_{6}^{+}$-valued second-order random field, on probability space $(\Theta, \mathcal{T}, \mathcal{P})$, indexed by $\mathbb{R}^{3}$, homogeneous, mean-square continuous, with mean value $[\mathbb{C}]=E\{[\mathbb{C}(\mathbf{x} ;[y])]\}$ that is therefore independent of $\mathbf{x}$ and $[y]$. Let $[\underline{\mathbb{L}}]$ be the upper triangular $(6 \times 6)$ real matrix such that $[\underline{\mathbb{C}}]=[\underline{\mathbb{L}}]^{T}[\underline{\mathbb{L}}]$. For fixed $[y]$, the normalized representation of $[\mathbb{C}(\mathbf{x} ;[y])]$ is written as,

$$
[\mathbb{C}(\mathbf{x} ;[y])]=\frac{1}{1+\epsilon}[\underline{\mathbb{}}]^{T}\left(\epsilon\left[I_{6}\right]+[\mathbf{C}(\mathbf{x} ;[y])]\right)[\underline{\mathbb{L}}],
$$


in which $\epsilon>0$ is given and where $\left\{[\mathbf{C}(\mathbf{x} ;[y])], \mathbf{x} \in \mathbb{R}^{3}\right\}$ is a $\mathbb{M}_{6}^{+}$-valued random field (by construction), defined on $(\Theta, \mathcal{T}, \mathcal{P})$, indexed by $\mathbb{R}^{3}$. Then $[\mathbf{C}(\cdot ;[y])]$ is homogeneous, mean-square continuous, and such that

$$
E\{[\mathbf{C}(\mathbf{x} ;[y])]\}=\left[I_{6}\right] \quad, \quad \forall \mathbf{x} \in \mathbb{R}^{3} .
$$

It should be noted that the lower bound $\epsilon[\underline{\mathbb{C}}] /(1+\epsilon)$ used in Eq. (4) could be replaced by a more general lower bound $\left[\mathbb{C}_{b}\right]$ in $\mathbb{M}_{6}^{+}$as proposed in $[48,58,61]$. Note also that the mean value $E\{[\widetilde{\mathbf{C}}(\mathbf{x})]\}$ of the normalized random apparent elasticity field $[\widetilde{\mathbf{C}}(\mathbf{x})\}=[\mathbf{C}(\mathbf{x} ;[\mathbf{Y}])]$ with uncertain spectral measure will not be equal to $\left[I_{6}\right]$ (that is not a difficulty) and we will have $E\{[\widetilde{\mathbf{C}}(\mathbf{x})]\} \simeq\left[I_{6}\right]$.

\subsection{Principle of construction of random field $[\boldsymbol{C}(\cdot ;[y])]$ given $[y]$}

By construction (see Section 4.1), $[\mathbf{C}(\cdot ;[y])]$ is a $\mathbb{M}_{6}^{+}$-valued random field indexed by $\mathbb{R}^{3}$ and homogeneous. For all $\mathbf{x}$ fixed in $\mathbb{R}^{3}$ and [y] fixed in $C_{y}$, the $\mathbb{M}_{6}^{+}$-valued random variable $[\mathbf{C}(\mathbf{x} ;[y])]$ is constructed by using the Maximum Entropy Principle under the following available information,

$$
E\{[\mathbf{C}(\mathbf{x} ;[y])]\}=\left[I_{6}\right], E\{\log (\operatorname{det}[\mathbf{C}(\mathbf{x} ;[y])])\}=b_{c},
$$

in which $b_{c}$ is independent of $\mathbf{x}$ and $[y]$ and such that $\left|b_{c}\right|<+\infty$. The second equality is introduced in order that the random matrix $[\mathbf{C}(\mathbf{x} ;[y])]^{-1}$ (that exists almost surely) be such that $E\left\{\left\|[\mathbf{C}(\mathbf{x} ;[y])]^{-1}\right\|_{2}^{2}\right\} \leq E\left\{\left\|[\mathbf{C}(\mathbf{x} ;[y])]^{-1}\right\|_{F}^{2}\right\}<+\infty$. With such a construction, $[\mathbf{C}(\mathbf{x} ;[y])]$ will appear as a nonlinear transformation of $6 \times(6+1) / 2=21$ independent normalized Gaussian real-valued random variables denoted by $\left\{G_{m n}(\mathbf{x} ;[y]), 1 \leq m \leq n \leq 6\right\}$ and such that

$$
E\left\{G_{m n}(\mathbf{x} ;[y])\right\}=0 \quad, \quad E\left\{G_{m n}(\mathbf{x} ;[y])^{2}\right\}=1 .
$$

The spatial correlation structure of random field $\left\{[\mathbf{C}(\mathbf{x} ;[y])], \mathbf{x} \in \mathbb{R}^{3}\right\}$ is introduced by considering 21 independent real-valued random fields $\left\{G_{m n}(\mathbf{x} ;[y]), \mathbf{x} \in \mathbb{R}^{3}\right\}$ for $1 \leq m \leq n \leq 6$, corresponding to 21 independent copies of a unique normalized Gaussian homogeneous mean-square continuous real-valued random field $\left\{G(\mathbf{x} ;[y]), \mathbf{x} \in \mathbb{R}^{3}\right\}$ given its normalized spectral measure parameterized by $[y]$. Note that the Gaussian random field $G(\cdot ;[y])$ is entirely defined by its normalized spectral measure (parameterized by $[y]$ ) because, for all $\mathbf{x}$ in $\mathbb{R}^{3}, E\{G(\mathbf{x} ;[y])\}=0$ and $E\left\{G(\mathbf{x} ;[y])^{2}\right\}=1$. The constant $b_{c}$ is eliminated in favor of a hyperparameter $\delta_{c}>0$, which allows for controlling the level of statistical fluctuations of $[\mathbf{C}(\mathbf{x} ;[y])]$, defined by

$$
\delta_{c}=\left(E\left\{\left\|[\mathbf{C}(\mathbf{x} ;[y])]-\left[I_{6}\right]\right\|_{F}^{2}\right\} / 6\right)^{1 / 2},
$$

which is independent of $\mathbf{x}$ and chosen independent of $[y]$.

\subsection{Construction of random field $[\boldsymbol{C}(\cdot ;[y])]$ given $[y]$}

The following construction of random field $[\mathbf{C}(\cdot ;[y])]$ given $[y]$ is adapted from the construction proposed in $[40,48,56,61]$ and is summarized below.

Probability distribution of the random matrix $[\mathbf{C}(\mathbf{x} ;[y])]$. Let $d^{S} C=2^{15 / 2} \prod_{1 \leq m \leq n \leq 6} d C_{m n}$ be the volume element on Euclidean space $\mathbb{M}_{6}^{S}$ in which $d C_{m n}$ is the Lebesgue measure on $\mathbb{R}$. For all $\mathbf{x}$ fixed in $\mathbb{R}^{3}$, the probability measure $P_{[\mathbf{C}(\mathbf{x} ;[y]]]}\left(d^{S} C\right)$ of the $\mathbb{M}_{6}^{+}$-valued random variable $[\mathbf{C}(\mathbf{x} ;[y])]$ constructed with the Maximum Entropy Principle under the constraints defined by Eq. (5), is 
independent of $\mathbf{x}$ (homogeneous random field), independent of $[y]$ (this marginal probability measure does not depend of the correlation structure), and can be found in [56].

Construction of a representation of random matrix $[\mathbf{C}(\mathbf{x} ;[y])]$. For all $\mathbf{x}$ fixed in $\mathbb{R}^{3}$ and $[y]$ fixed in $C_{y}$, random matrix $[\mathbf{C}(\mathbf{x} ;[y])]$ is written as

$$
[\mathbf{C}(\mathbf{x} ;[y])]=[\mathbf{L}(\mathbf{x} ;[y])]^{T}[\mathbf{L}(\mathbf{x} ;[y])],
$$

in which $[\mathbf{L}(\mathbf{x} ;[y])]$ is an upper triangular random matrix in $\mathbb{M}_{6}$ for which its entries are defined as follows. The 21 random variables $\left\{[\mathbf{L}(\mathbf{x} ;[y])]_{m n}, 1 \leq m \leq n \leq 6\right\}$ are mutually independent. For $1 \leq m<n \leq 6$, we have

$$
[\mathbf{L}(\mathbf{x} ;[y])]_{m n}=\sigma_{c} G_{m n}(\mathbf{x} ;[y]),
$$

with $\sigma_{c}=\delta_{c} / \sqrt{7}$ and where $G_{m n}(\mathbf{x} ;[y])$ is a normalized Gaussian real-valued random variable (see Eq. (6)). For $1 \leq m=n \leq 6$, we have

$$
[\mathbf{L}(\mathbf{x} ;[y])]_{m m}=\sigma_{c} \sqrt{2 h\left(G_{m m}(\mathbf{x} ;[y]) ; \alpha_{m}\right)},
$$

in which $\alpha_{m}=1 /\left(2 \sigma_{c}^{2}\right)+(1-m) / 2$ such that $\alpha_{1}>\ldots>\alpha_{6}>3$ and where $G_{m m}(\mathbf{x} ;[y])$ is a normalized Gaussian real-valued random variable (see Eq. (6)). The function $b \mapsto h(b ; \alpha)$ is such that $\Gamma_{\alpha}=h(\mathcal{N} ; \alpha)$ is a Gamma random variable with parameter $\alpha$ when $\mathcal{N}$ is the normalized Gaussian real-valued random variable. Hyperparameter $\delta_{c}$ must belong to the real interval $] 0, \sqrt{7 / 11}[$.

Construction of the family of random fields $\left\{G_{m n}(\cdot ;[y])\right\}$. The 21 random fields $\left\{G_{m n}(\mathbf{x} ;[y]), \mathbf{x} \in\right.$ $\left.\mathbb{R}^{3}\right\}$ for $1 \leq m \leq n \leq 6$ are 21 independent copies of a normalized Gaussian homogeneous mean-square continuous real-valued random field $\left\{G(\mathbf{x} ;[y]), \mathbf{x} \in \mathbb{R}^{3}\right\}$,

$$
E\{G(\mathbf{x} ;[y])\}=0, E\left\{G(\mathbf{x} ;[y])^{2}\right\}=1, \forall \mathbf{x} \in \mathbb{R}^{3},
$$

and which will be defined in Section 4.4 for imposing its spatial correlation structure via its spectral measure. parameterized by $[y]$.

\subsection{Construction of the random field $G(\cdot ;[y])$ with uncertain spectral measure parameterized by $[y]$}

In the following, we will introduce a nominal value $[y]$ of $[y]$. In order to simplify the notation, for $[y]=[y]$, the random field $\left\{G(\mathbf{x} ;[\underline{y}]), \mathbf{x} \in \mathbb{R}^{3}\right\}$ will simply be denoted by $\{G(\mathbf{x}), \mathbf{x} \in$ $\left.\mathbb{R}^{3}\right\}$, that is to say, $\bar{G}=G(\cdot ;[y])$.

In this section, we construct the normalized Gaussian, homogeneous, second-order, meansquare continuous random field $\left\{G(\mathbf{x}), \mathbf{x} \in \mathbb{R}^{3}\right\}$. Therefore, there exists a positive bounded spectral measure $m_{G}(d \mathbf{k})$ on $\mathbb{R}^{3}$ such that the correlation function $r_{G}(\zeta)=E\{G(\mathbf{x}+\zeta) G(\mathbf{x})\}$ of $G$ is written, for all $\mathbf{x}$ and $\zeta$ in $\mathbb{R}^{3}$, as

$$
r_{G}(\zeta)=\int_{\mathbb{R}^{3}} e^{\iota \mathbf{k} \cdot \zeta} m_{G}(d \mathbf{k})=\int_{\mathbb{R}^{3}} \cos (\mathbf{k} \cdot \zeta) m_{G}(d \mathbf{k}),
$$

in which $\iota=\sqrt{-1}$, where $\mathbf{k} \cdot \zeta=\sum_{j=1}^{3} k_{j} \zeta_{j}$, and where $d \mathbf{k}=d k_{1} d k_{2} d k_{3}$. 


\subsubsection{Spectral density function and spatial correlation lengths of random field $G$}

Let us assume that $m_{G}(d \mathbf{k})=s(\mathbf{k}) d \mathbf{k}$ admits a spectral density function $\mathbf{k} \mapsto s(\mathbf{k})$ from $\mathbb{R}^{3}$ into $\mathbb{R}^{+}$. Equations (10) and (11) yield $r_{G}(0)=E\left\{G(\mathbf{x})^{2}\right\}=1$, and consequently, $\int_{\mathbb{R}^{3}} s(\mathbf{k}) d \mathbf{k}=1$. In addition, it is assumed that $s$ has a compact support $\overline{\mathbb{K}}=\partial \mathbb{K} \cup \mathbb{K}$ with $\left.\mathbb{K}=\prod_{j=1}^{3}\right]-K_{j}, K_{j}[$ in which $K_{j} \in\left[K_{j}^{\min }, K_{j}^{\max }\right]$ with $0<K_{j}^{\min }<K_{j}^{\max }<+\infty$, and that $s$ is a continuous function on $\mathbb{R}^{3}$. Since supp $s=\overline{\mathbb{K}}$, we must have $s(\mathbf{k})=0$ for all $\mathbf{k}$ in $\partial \mathbb{K}$. We have

$$
\int_{\mathbb{R}^{3}} s(\mathbf{k}) d \mathbf{k}=\int_{\overline{\mathbb{K}}} s(\mathbf{k}) d \mathbf{k}=1
$$

and $s(-\mathbf{k})=s(\mathbf{k})$ for all $\mathbf{k}$ in $\mathbb{R}^{3}$ because random field $G$ is real. In addition to this symmetry property, it is assumed that $s$ satisfies the quadrant symmetry [52] that is defined as follows. Let $\mathbf{k}_{\{-j\}}$ be a vector $\mathbf{k}$ for which its component $k_{j}$ is replaced by $-k_{j}$. Then for all $\mathbf{k}$ in $\mathbb{R}^{3}$ and for all $j \in\{1,2,3\}$, the quadrant symmetry of $s$ means that $s\left(\mathbf{k}_{\{-j\}}\right)=s(\mathbf{k})$. The quadrant symmetry is often of practical importance in terms of modeling. This type of symmetry occurs naturally in many models of covariance function used for the applications such as for the isotropic, spherical, radial, ellipsoidal covariance functions and for a separable correlation structure.

The spatial correlation length (without uncertainties in the spectral measure) for coordinate $\zeta_{j}$ is defined by

$$
L_{c j}=\int_{0}^{+\infty}\left|r_{G}\left(0, \ldots, \zeta_{j}, \ldots, 0\right)\right| d \zeta_{j},
$$

and is assumed to be finite.

\subsubsection{Definition of the spectral domain sampling}

Let $v_{s}$ be a given even integer. For $j$ in $\{1,2,3\}$, we define $\Delta_{j}=2 K_{j} / v_{s}$ as the sampling step of interval $\left[-K_{j}, K_{j}\right]$ and we introduce its spectral sampling points,

$$
k_{j \beta_{j}}=-K_{j}+\left(\beta_{j}-1 / 2\right) \Delta_{j} \quad, \quad \beta_{j}=1, \ldots, v_{s} .
$$

Let $\mathcal{B}$ be the finite subset of $\mathbb{N}^{3}$ such that

$$
\mathcal{B}=\left\{\boldsymbol{\beta}=\left(\beta_{1}, \beta_{2}, \beta_{3}\right), \beta_{j}=1, \ldots, v_{s} \text { for } j=1,2,3\right\} .
$$

Let $\Delta, K, v$ be such that $\Delta=\Delta_{1} \Delta_{2} \Delta_{3}, K=K_{1} K_{2} K_{3}, v=\left(v_{s}\right)^{3}$, and let $\mathbf{k}_{\beta}$ be such that

$$
\forall \boldsymbol{\beta} \in \mathcal{B}, \mathbf{k}_{\boldsymbol{\beta}}=\left(k_{1 \beta_{1}}, k_{2 \beta_{2}}, k_{3 \beta_{3}}\right) \in \mathbb{K} \subset \mathbb{R}^{3} .
$$

\subsubsection{Discretization of the spectral measure and convergence properties}

Let $\delta_{\mathbf{k}_{\beta}}(\mathbf{k})=\otimes_{j=1}^{3} \delta_{k_{j \beta_{j}}}\left(k_{j}\right)$ be the Dirac measure on $\mathbb{R}^{3}$ at sampling point $\mathbf{k}_{\beta} \in \mathbb{K} \subset \mathbb{R}^{3}$ defined in Section 4.4.2. Let $m_{G}^{v}(d \mathbf{k})$ be the positive bounded measure on $\mathbb{R}^{3}$ defined by

$$
m_{G}^{v}(d \mathbf{k})=\sum_{\beta \in \mathcal{B}} s_{\beta}^{\Delta} \delta_{\mathbf{k}_{\beta}}(\mathbf{k}) \quad, \quad s_{\beta}^{\Delta}=\Delta s\left(\mathbf{k}_{\beta}\right)
$$

which is such that $m_{G}^{v}\left(\mathbb{R}^{3}\right)=\sum_{\beta \in \mathcal{B}} s_{\beta}^{\Delta}=\eta_{v}$ with $\eta_{v}>0$. The sequence of measures $\left\{m_{G}^{v}(d \mathbf{k})\right\}_{v}$ converges narrowly towards the measure $m_{G}(d \mathbf{k})$ and the positive sequence $\left\{\eta_{v}\right\}_{v}$ converges towards $1[62,64]$. 
In the following, it is assumed that $v=\left(v_{s}\right)^{3}$ is chosen sufficiently large in order that $\left|\sum_{\beta \in \mathcal{B}} s_{\beta}^{\Delta}-1\right| \leq \epsilon_{s} \ll 1$ and consequently, we will write

$$
\sum_{\beta \in \mathcal{B}} s_{\beta}^{\Delta} \simeq 1
$$

\subsubsection{Definition of the dimensionless spectral density function}

For all $\mathbf{k}$ in $\mathbb{R}^{3}$, the spectral density function $\mathbf{k} \mapsto s(\mathbf{k})$ (defined in Section 4.4.1) is written as

$$
s\left(k_{1}, k_{2}, k_{3}\right)=\left(K_{1} K_{2} K_{3}\right)^{-1} \chi\left(\frac{k_{1}}{K_{1}}, \frac{k_{2}}{K_{2}}, \frac{k_{3}}{K_{3}}\right),
$$

with $\chi$ a given function $\kappa=\left(\kappa_{1}, \kappa_{2}, \kappa_{3}\right) \mapsto \chi\left(\kappa_{1}, \kappa_{2}, \kappa_{3}\right)$ from $\mathbb{R}^{3}$ into $\mathbb{R}^{+}$with compact support $[-1,1]^{3}$. Function $\chi$ has the same properties as $s$, that is to say, $\chi(-\kappa)=\chi(\kappa)$, quadrant symmetry, and continuity. For $j=1,2,3$, the change of variable $\kappa_{j}=k_{j} / K_{j}$ yields

$$
s(\mathbf{k}) d \mathbf{k}=\chi(\boldsymbol{\kappa}) d \boldsymbol{\kappa},
$$

and thus $m_{G}(d \mathbf{k})=\mu_{G}(d \boldsymbol{\kappa})$ with $\mu_{G}(d \boldsymbol{\kappa})=\chi(\boldsymbol{\kappa}) d \boldsymbol{\kappa}$. Therefore, Eq. (12) yields

$$
\int_{\mathbb{R}^{3}} \chi(\boldsymbol{\kappa}) d \boldsymbol{\kappa}=\int_{[-1,1]^{3}} \chi(\boldsymbol{\kappa}) d \boldsymbol{\kappa}=1 .
$$

The dimensionless spectral domain sampling is directly deduced from Section 4.4.2,

$$
\left.\forall \boldsymbol{\beta} \in \mathcal{B}, \kappa_{\beta}=\left(\kappa_{\beta_{1}}, \kappa_{\beta_{2}}, \kappa_{\beta_{3}}\right) \in\right]-1,1\left[^{3} \subset \mathbb{R}^{3},\right.
$$

in which for $j \in\{1,2,3\}$, we have

$$
\kappa_{\beta_{j}}=-1+\left(\beta_{j}-\frac{1}{2}\right) \frac{2}{v_{s}} \quad, \quad \beta_{j} \in\left\{1, \ldots, v_{s}\right\} .
$$

The discretization $\mu_{G}^{v}(d \boldsymbol{\kappa})$ of $\mu_{G}(d \boldsymbol{\kappa})$, such that $\mu_{G}^{v}(d \boldsymbol{\kappa})=m_{G}^{v}(d \mathbf{k})$, is written as

$$
\mu_{G}^{v}(d \boldsymbol{\kappa})=\sum_{\beta \in \mathcal{B}} \chi_{\beta}^{\Delta} \delta_{\boldsymbol{\kappa}_{\beta}}(\boldsymbol{\kappa}), \chi_{\boldsymbol{\beta}}^{\Delta}=\left(2 / v_{s}\right)^{3} \chi\left(\boldsymbol{\kappa}_{\boldsymbol{\beta}}\right),
$$

in which $\delta_{\kappa_{\beta}}=\otimes_{j=1}^{3} \delta_{\kappa_{\beta}}\left(\kappa_{j}\right)$ and where, from Eq. (15),

$$
\sum_{\beta \in \mathcal{B}} \chi_{\beta}^{\Delta} \simeq 1
$$

It can be seen that measure $\mu_{G}^{v}(d \kappa)$ is independent of $K_{1}, K_{2}$, and $K_{3}$. In order to introduce the probability model of uncertainties in the spectral measure, we define hereinafter an adapted parameterization $[y]$ that takes into account quadrant symmetry. 


\subsubsection{Parameterization of the discretized dimensionless spectral measure}

Let $\widehat{v}_{s}=v_{s} / 2$ in which $v_{s}$ is an even integer. Let $C_{y}$ be the subset of $\mathbb{M}_{3, \widehat{v}_{s}}$ defined by

$$
C_{y}=\left\{[y] \in \mathbb{M}_{3, \widehat{v}_{s}},[y]_{\widehat{j} \widehat{\beta}} \in[0,1], \forall j, \forall \widehat{\beta}\right\},
$$

in which $j \in\{1,2,3\}$ and $\widehat{\beta} \in\left\{1, \ldots, \widehat{v}_{s}\right\}$. Let $[\underline{y}]$ be in $C_{y}$ such that

$$
\left[\underline{y}_{\widehat{j} \widehat{\beta}}=1 / 2, \forall j \in\{1,2,3\}, \forall \widehat{\beta} \in\left\{1, \ldots, \widehat{v}_{s}\right\} .\right.
$$

We now introduce the subset $\widehat{\mathcal{B}} \subset \mathcal{B} \subset \mathbb{N}^{3}$ such that

$$
\widehat{\mathcal{B}}=\left\{\widehat{\boldsymbol{\beta}}=\left(\widehat{\beta}_{1}, \widehat{\beta}_{2}, \widehat{\beta}_{3}\right), \widehat{\boldsymbol{\beta}}_{j}=1, \ldots, \widehat{v}_{s} \text { for } j=1,2,3\right\},
$$

which has $\widehat{v}=\left(\widehat{v}_{s}\right)^{3}=v / 8$ elements. We define the finite family of functions $[y] \mapsto a_{\widehat{\boldsymbol{\beta}}}([y])$ from $C_{y}$ into $\mathbb{R}$ such that, for all $\widehat{\boldsymbol{\beta}}$ in $\widehat{\mathcal{B}}$,

$$
a_{\widehat{\beta}}([y])=\sqrt{\chi_{\widehat{\beta}}^{\Delta}} q_{\widehat{\beta}}\left([y] ; \delta_{s}\right),
$$

in which $\delta_{s}>0$ is a hyperparameter that will allow the level of spectrum uncertainties to be controlled and where $[y] \mapsto q_{\widehat{\beta}}\left([y] ; \delta_{s}\right)$ is any given continuous real function on $C_{y}$ such that

$$
q_{\widehat{\beta}}\left([\underline{y}] ; \delta_{s}\right)=1 .
$$

For all $[y]$ in $C_{y}$, let $\left\{a_{\beta}([y]), \beta \in \mathcal{B}\right\}$ be the $v$ real numbers that are directly constructed from $\left\{a_{\widehat{\boldsymbol{\beta}}}([y]), \widehat{\boldsymbol{\beta}} \in \widehat{\mathcal{B}}\right\}$ using the quadrant symmetry (see Section 4.4.1). An example of such a construction is given in Section 6.1. For all $\boldsymbol{\beta}$ in $\mathcal{B}$, we define the function $[y] \mapsto \widetilde{\chi}_{\beta}^{\Delta}([y])$ from $C_{y}$ into $\mathbb{R}^{+}$such that

$$
\widetilde{\chi}_{\beta}^{\Delta}([y])=a_{\beta}([y])^{2}\left(\sum_{\beta^{\prime} \in \mathcal{B}} a_{\beta^{\prime}}([y])^{2}\right)^{-1} .
$$

The dimensionless spectral measure $\widetilde{\mu}_{G}^{v}(d \boldsymbol{\kappa} ;[y])$ for $[y]$ given in $C_{y}$ is then defined by

$$
\widetilde{\mu}_{G}^{v}(d \kappa ;[y])=\sum_{\beta \in \mathcal{B}} \widetilde{\chi}_{\beta}^{\Delta}([y]) \delta_{\kappa_{\beta}}(\kappa) .
$$

\subsubsection{Random discretized dimensionless spectral measure}

Using Sections 4.4.4 and 4.4.5, and assuming that Eq. (18) holds, it can then easily be proven the following results.

(i) For all $\boldsymbol{\beta}$ in $\mathcal{B}$, function $[y] \mapsto \widetilde{\chi}_{\beta}^{\Delta}([y])$ is continuous on $C_{y}$, such that $\widetilde{\chi}_{\boldsymbol{\beta}}^{\Delta}([y]) \simeq \chi_{\beta}^{\Delta}$, and we have

$$
\forall[y] \in C_{y} \quad, \quad \sum_{\beta \in \mathcal{B}} \widetilde{\chi}_{\beta}^{\Delta}([y])=1 .
$$

(ii) Let $[\mathbf{Y}]$ be the $\mathbb{M}_{3, \widehat{v}_{s}}$-valued random variable, defined on $(\Theta, \mathcal{T}, \mathcal{P})$, whose support of its probability measure is $C_{y} \subset \mathbb{M}_{3, \widehat{v}_{s}}$, and such that $\left\{[\mathbf{Y}]_{\widehat{j} \widehat{\beta}}, j \in\{1,2,3\}, \widehat{\beta} \in\left\{1, \ldots, \widehat{v}_{s}\right\}\right\}$ are $3 \widehat{v}_{s}$ independent uniform random variables on $[0,1]$. Its mean value is

$$
E\{[\mathbf{Y}]\}=\int_{C_{y}}[y] P_{[\mathbf{Y}]}(d y)=\int_{C_{y}}[y] d y=[\underline{y}] .
$$


For all $\widehat{\boldsymbol{\beta}}$ in $\widehat{\mathcal{B}}, A_{\widehat{\boldsymbol{\beta}}}=a_{\widehat{\boldsymbol{\beta}}}([\mathbf{Y}])$ is a second-order real-valued random variable.

(iii) For all $\beta$ in $\mathcal{B}, \widetilde{\chi}_{\beta}^{\Delta}([\mathbf{Y}])$ is a second-order positive-valued random variable, defined on $(\Theta, \mathcal{T}, \mathcal{P})$ such that

$$
\sum_{\beta \in \mathcal{B}} \widetilde{\chi}_{\beta}^{\Delta}([\mathbf{Y}])=1 \quad \text { a.s } .
$$

(iv) For given $[y]$ in $C_{y}$, the dimensionless spectral measure $\widetilde{\mu}_{G}^{v}(d \boldsymbol{\kappa}$; $[y])$ is a bounded positive measure on $\mathbb{R}^{3}$, is such that $\left.\widetilde{\mu}_{G}^{v}(d \boldsymbol{\kappa} ; \underline{y}]\right) \simeq \mu_{G}^{v}(d \boldsymbol{\kappa})$, and we have

$$
\forall[y] \in C_{y} \quad, \quad \widetilde{\mu}_{G}^{v}\left(\mathbb{R}^{3} ;[\underline{y}]\right)=\sum_{\beta \in \mathcal{B}} \widetilde{\chi}_{\beta}^{\Delta}([\underline{y}])=1 .
$$

\subsection{Construction of the normalized Gaussian random field $G^{v}(\cdot ;[y])$ given $[y]$ in $C_{y}$}

Let $v=\left(v_{s}\right)^{3}$ be fixed. Let $\left\{Z_{\beta}, \boldsymbol{\beta} \in \mathcal{B}\right\}$ and $\left\{\Phi_{\beta}, \boldsymbol{\beta} \in \mathcal{B}\right\}$ be $2 v$ independent random variables defined on $(\Theta, \mathcal{T}, \mathcal{P})$, which are independent of $[\mathbf{Y}]$. For $\beta$ in $\left.\mathcal{B}, Z_{\beta}=\sqrt{-\log \left(\Psi_{\beta}\right.}\right)$ in which $\Psi_{\beta}$ is uniform of $[0,1]$ and $\Phi_{\beta}$ is uniform on $[0,2 \pi]$. Let $P_{\mathbf{Z}}(d \mathbf{z})$ and $P_{\boldsymbol{\Phi}}(d \boldsymbol{\varphi})$ be the probability measures on $\mathbb{R}^{v}$ of the $\mathbb{R}^{v}$-valued random variables $\mathbf{Z}=\left\{Z_{\beta}, \boldsymbol{\beta} \in \mathcal{B}\right\}$ and $\mathbf{\Phi}=\left\{\Phi_{\beta}, \boldsymbol{\beta} \in \mathcal{B}\right\}$. The unbounded support $C_{z}$ of $P_{\mathbf{Z}}(d \mathbf{z})$ is

$$
C_{z}=\left\{\mathbf{z}=\left\{z_{\beta}, \boldsymbol{\beta} \in \mathcal{B}\right\}, z_{\beta}>0\right\} \subset \mathbb{R}^{v}
$$

and the compact support $C_{\varphi}$ of $P_{\boldsymbol{\Phi}}(d \varphi)$ is

$$
C_{\varphi}=\left\{\varphi=\left\{\varphi_{\beta}, \boldsymbol{\beta} \in \mathcal{B}\right\}, \varphi_{\beta} \in[0,2 \pi]\right\} \subset \mathbb{R}^{\nu} .
$$

We define the mapping $\mathbf{x} \mapsto g^{\nu}(\mathbf{x} ;[y], \mathbf{z}, \varphi)$ from $\mathbb{R}^{3}$ into $\mathbb{R}$ be such that, for all $\{[y], \mathbf{z}, \varphi\}$ in $\mathcal{C}_{y} \times \mathcal{C}_{z} \times \mathcal{C}_{\varphi}$,

$$
\begin{aligned}
& g^{v}(\mathbf{x} ;[y], \mathbf{z}, \boldsymbol{\varphi})= \\
& \quad \sum_{\beta \in \mathcal{B}} \sqrt{2 \widetilde{\chi}_{\beta}^{\Delta}([y])} z_{\beta} \cos \left(\varphi_{\beta}+\sum_{j=1}^{3} K_{j} \kappa_{\beta_{j}} x_{j}\right) .
\end{aligned}
$$

For all $[y]$ in $C_{y}$, we define the real-valued random field $\left\{G^{v}(\mathbf{x} ;[y]), \mathbf{x} \in \mathbb{R}^{3}\right\}$ such that

$$
G^{v}(\mathbf{x} ;[y])=g^{v}(\mathbf{x} ;[y], \mathbf{Z}, \mathbf{\Phi}) .
$$

For all $[y]$ in $C_{y}$, the real-valued random field $\left\{G^{v}(\mathbf{x} ;[y]), \mathbf{x} \in \mathbb{R}^{3}\right\}$ is Gaussian, homogeneous, second-order, mean-square continuous [65, 64], and normalized,

$$
E\left\{G^{v}(\mathbf{x} ;[y])\right\}=0, E\left\{G^{v}(\mathbf{x} ;[y])^{2}\right\}=1, \forall \mathbf{x} \in \mathbb{R}^{3} .
$$

Its dimensionless spectral measure $\widetilde{\mu}_{G}^{v}(d \boldsymbol{\kappa} ;[y])$, expressed with the dimensionless spectral variable $\kappa=\left(\kappa_{1}, \kappa_{2}, \kappa_{3}\right)$ with $\kappa_{j}=k_{j} / K_{j}$, is the spectral measure defined by Eq. (21). 


\subsection{Correlation function of the normalized Gaussian random field $G^{v}(\cdot ;[y])$ given $[y]$}

Let $[y]$ be fixed in $C_{y}$. Using Eqs. (23) with (22) and since $E\left\{Z_{\beta}^{2}\right\}=1, E\left\{\cos \left(\varphi_{\beta}+y\right)\right\}=0$, and $E\left\{\cos \left(\varphi_{\beta}+y\right) \cos \left(\varphi_{\beta^{\prime}}+y^{\prime}\right)\right\}=\delta_{\beta \beta^{\prime}} \cos \left(y-y^{\prime}\right)$, it can be deduced that the correlation function $\boldsymbol{\zeta} \mapsto \vec{r}_{G}^{v}(\zeta ;[y])=E\left\{G^{\nu}(\mathbf{x}+\zeta ;[y]) G^{v}(\mathbf{x} ;[y])\right\}$ of random field $\left\{G^{v}(\mathbf{x} ;[y]), \mathbf{x} \in \mathbb{R}^{3}\right\}$ is written, for all $\zeta=\left(\zeta_{1}, \zeta_{2}, \zeta_{3}\right) \in \mathbb{R}^{3}$, as

$$
\widetilde{r}_{G}^{v}(\zeta ;[y])=\sum_{\boldsymbol{\beta} \in \mathcal{B}} \widetilde{\chi}_{\boldsymbol{\beta}}^{\Delta}([y]) \cos \left(\sum_{j=1}^{3} K_{j} \kappa_{\beta_{j}} \zeta_{j}\right) .
$$

For $j \in\{1,2,3\}$, the change of variable $\zeta_{j}=\xi_{j} / K_{j}$ in the right-hand side of Eq. (24) yields the dimensionless correlation function that is written, for all $\boldsymbol{\xi}=\left(\xi_{1}, \xi_{2}, \xi_{3}\right)$, as

$$
\widetilde{\rho}_{G}^{v}(\boldsymbol{\xi} ;[y])=\sum_{\boldsymbol{\beta} \in \mathcal{B}} \widetilde{\chi}_{\boldsymbol{\beta}}^{\Delta}([y]) \cos \left(\sum_{j=1}^{3} \kappa_{\beta_{j}} \xi_{j}\right) .
$$

It can easily be seen that $\widetilde{\rho}_{G}^{v}(\xi ;[y])$ defined by Eq. (25) is associated with the the dimensionless spectral measure $\widetilde{\mu}_{G}^{v}(d \kappa ;[y])$ defined by Eq. (21).

4.7. Non-Gaussian random field $[\mathbb{C}(\cdot ;[y])]$ parameterized by $[y]$ and random field $[\widetilde{\mathbb{C}}]$ with uncertain spectral measure

The non-Gaussian random field $\mathbf{C}(\cdot,[y])$ given $[y]$ in $C_{y}$ is defined by Eqs. (8) to (9) in which the 21 Gaussian random fields $\left\{G_{m n}(\mathbf{x} ;[y]), \mathbf{x} \in \mathbb{R}^{3}\right\}_{1 \leq m \leq n \leq 6}$ are replaced by 21 independent copies of the Gaussian real-valued random field $\left\{G^{v}(\mathbf{x} ;[y]), \mathbf{x} \in \mathbb{R}^{3}\right\}$ defined by Eq. (23), and denoted by $\left\{G_{m n}^{v}(\mathbf{x} ;[y]), \mathbf{x} \in \mathbb{R}^{3}\right\}_{1 \leq m \leq n \leq 6}$. For all $[y]$ in $C_{y}, \mathbf{x}$ in $\mathbb{R}^{3}$, and for $1 \leq m \leq n \leq 6$, using Eq. (23) yields

$$
G_{m n}^{v}(\mathbf{x} ;[y])=g^{v}\left(\mathbf{x} ;[y], \mathbf{Z}^{m n}, \boldsymbol{\Phi}^{m n}\right),
$$

in which $\left\{\mathbf{Z}^{m n}, \boldsymbol{\Phi}^{m n}\right\}_{1 \leq m \leq n \leq 6}$ are 21 independent copies of $\mathbb{R}^{v}$-valued random variables $\mathbf{Z}$ and $\boldsymbol{\Phi}$ (see Section 4.5), and we have

$$
E\left\{G_{m n}^{v}(\mathbf{x} ;[y])\right\}=0 \quad, \quad E\left\{G_{m n}^{v}(\mathbf{x} ;[y])^{2}\right\}=1 .
$$

It is assumed that $v$ is fixed as explained in Section 4.4.3. The random field $\left\{[\widetilde{\mathbb{C}}(\mathbf{x})] \in \mathbb{R}^{3}\right\}$ with uncertain spectral measure is defined by

$$
[\widetilde{\mathbb{C}}(\mathbf{x})]=[\mathbb{C}(\mathbf{x} ;[\mathbf{Y}])], \quad \forall \mathbf{x} \in \mathbb{R}^{3},
$$

in which [Y] is the $\mathbb{M}_{3, \widehat{v}_{s}}$ - valued random variable defined in Section 4.4.6-(ii).

4.8. Remark concerning the principle used for the construction of random field $[\boldsymbol{C}(\cdot ;[y])]$ given $[y]$

For given $[y]$, we have seen in Sections 4.2 and 4.3 that random field $[\mathbf{C}(\cdot ;[y])]$ depends on hyperparameter $\delta_{c}$ and on 21 independent copies $\left\{G_{m n}(\mathbf{x} ;[y]), \mathbf{x} \in \mathbb{R}^{3}\right\}_{1 \leq m \leq n \leq 6}$ of a unique normalized Gaussian homogeneous mean-square continuous real-valued random field $\{G(\mathbf{x} ;[y]), \mathbf{x} \in$ $\mathbb{R}^{3}$ \} for which its normalized spectral measure is $m_{G}(d \mathbf{k})=s(\mathbf{k}) d \mathbf{k}$ (parameterized by [y]). We have also seen that function $s$ was defined by three parameters $K_{1}, K_{2}, K_{3}$ that control the compact support of $s$ (see Section 4.4.1) and by the dimensionless spectral density function $\kappa \mapsto \chi(\kappa)$ 
(see Section 4.4.4). Finally, since random field $[\mathbf{C}(\cdot ;[y])$ is a nonlinear transformation of 21 realvalued random fields $\left\{G_{m n}(\mathbf{x} ;[y]), \mathbf{x} \in \mathbb{R}^{3}\right\}_{1 \leq m \leq n \leq 6}$ (see Sections 4.3 and 4.4), it can be deduced that the tensor-valued covariance function of random field $[\mathbf{C}(\cdot ;[y])$ given $[y]$ is controlled by the scalar parameters $\delta_{c}, K_{1}, K_{2}, K_{3}$ and by the positive-valued function $\kappa \mapsto \chi(\kappa)$ on $[-1,1]^{3}$. For given $[y]$, these parameters and this function can be estimated by solving a statistical inverse problem for which experimental observations (targets) related to the stochastic BVP would be given. For improving such an indirect representation of the tensor-valued covariance function of random field $[\mathbf{C}(\cdot ;[y])$ given $[y]$, the number of parameters could be increased as follows. Instead of constructing random fields $\left\{G_{m n}(\mathbf{x} ;[y]), \mathbf{x} \in \mathbb{R}^{3}\right\}_{1 \leq m \leq n \leq 6}$ as 21 independent copies of random field $\left\{G(\mathbf{x} ;[y]), \mathbf{x} \in \mathbb{R}^{3}\right\}$, we could directly construct the 21 independent random fields $\left\{G_{m n}(\mathbf{x} ;[y]), \mathbf{x} \in \mathbb{R}^{3}\right\}_{1 \leq m \leq n \leq 6}$, each random field $\left\{G_{m n}(\mathbf{x} ;[y]), \mathbf{x} \in \mathbb{R}^{3}\right\}$ having its own compact spectral density function $\mathbf{k} \mapsto s^{m n}(\mathbf{k})$ defined by three parameters $K_{1}^{m n}, K_{2}^{m n}, K_{3}^{m n}$ that would control its support and by the positive-valued function $\boldsymbol{\kappa} \mapsto \chi^{m n}(\boldsymbol{\kappa})$ on $[-1,1]^{3}$. In such a case, all the presented developments for the uncertain spectral measure could directly be extended without any difficulty. Given $[y]$, the tensor-valued covariance function of random field $[\mathbf{C}(\cdot ;[y])$ would be controlled by 64 parameters $\delta_{c},\left\{K_{1}^{m n}, K_{2}^{m n}, K_{3}^{m n}, 1 \leq m \leq n \leq 6\right\}$ and by 21 functions $\left\{\chi^{m n}, 1 \leq m \leq n \leq 6\right\}$, which would considerably increase the potentiality of the constructed representation.

\section{Solution of the stochastic boundary value problem and random eigenvalues of the ran- dom effective elasticity matrix}

Strong stochastic solution of the weak formulation. We now consider the nonhomogeneous Dirichlet stochastic Boundary Value Problem (BVP) defined by Eqs. (1) to (3), in which [y] is modeled by the $\mathbb{M}_{3, \widehat{v}_{s}}$-valued random variable [Y] , and for which the probabilistic model of the random field $\left\{[\widetilde{\mathbb{C}}(\mathbf{x})]=[\mathbb{C}(\mathbf{x} ;[\mathbf{Y}])], \mathbf{x} \in \mathbb{R}^{3}\right\}$ with uncertain spectral measure is the one defined in Section 4. A finite element approximation of the weak formulation of this stochastic BVP is constructed in order to perform the computational stochastic homogenization. On the other hand, the Monte Carlo simulation method is used as stochastic solver. Consequently, we need not to construct and to analyze a weak stochastic solution of the weak formulation of this stochastic BVP, but we only need to construct and to analyze the strong stochastic solution of this weak formulation of the stochastic BVP. In [62], it is mathematically proven that, for $1 \leq \ell \leq r \leq 3$, there is a unique strong stochastic solution solution $\left\{\widetilde{\mathbf{U}}^{\ell r}(\mathbf{x})=\mathbf{u}^{\ell r}(\mathbf{x} ;[\mathbf{Y}]), \mathbf{x} \in \bar{\Omega}\right\}$ of the weak formulation, which is a second-order random field, that is to say, such that $E\left\{\left\|\widetilde{\mathbf{U}}^{\ell r}(\mathbf{x})\right\|_{2}^{2}\right\}=\underline{\gamma}_{u}^{2}<+\infty$ for all $\mathbf{x}$ in $\bar{\Omega}$, in which the constant $\underline{\gamma}_{u}^{2}$ is independent of $\mathbf{x}$ and whose expression is detailed in [62].

Random eigenvalues of the random effective elasticity matrix. The random effective elasticity matrix $\left[\widetilde{\mathbb{C}}^{\text {eff }}\right]$, which corresponds to the random apparent elasticity field $\widetilde{\mathbb{C}}$ for which its spectral measure is uncertain, can then be written as $\left[\widetilde{\mathbb{C}}^{\text {eff }}\right]=\left[\mathbb{C}^{\text {eff }}([\mathbf{Y}])\right]$. Let $\widetilde{\Lambda}_{1} \geq \widetilde{\Lambda}_{2} \geq \ldots \geq \widetilde{\Lambda}_{6}$ be the ordered (a.s) random eigenvalues of $\left[\widetilde{\mathbb{C}}^{\text {eff }}\right]$. Let $\widetilde{\boldsymbol{\Lambda}}=\left(\widetilde{\Lambda}_{1}, \ldots, \widetilde{\Lambda}_{6}\right)$ be the $\mathbb{R}^{6}$-valued random variable whose support of its probability measure $P_{\widetilde{\Lambda}}(\widetilde{d})$ is $\left(\mathbb{R}^{+*}\right)^{6}$. The operator norm of $\left[\widetilde{\mathbb{C}}^{\text {eff }}\right]$ is $\left\|\left[\widetilde{\mathbb{C}}^{\text {eff }}\right]\right\|_{2}=\widetilde{\Lambda}_{1}$. It is also mathematically proven in [62] that $\widetilde{\Lambda}$ is a second-order $\mathbb{R}^{6}$-valued random variable, that is to say, $E\left\{\|\widetilde{\boldsymbol{\Lambda}}\|_{2}^{2}\right\}<+\infty$. 


\section{Data and models for performing the computational stochastic homogenization}

6.1. Defining the uncertain spectral measure based on a separable spatial correlation structure

The computational stochastic homogenization analysis that we will perform is based on the following separable spatial correlation structure of the spectral measure.

Spectral density function. For all $\mathbf{k}=\left(k_{1}, k_{2}, k_{3}\right)$ in $\mathbb{R}^{3}$, the spectral density function is written as $s(\mathbf{k})=\prod_{j=1}^{3} s_{j}\left(k_{j}\right)$ in which, for $j \in\{1,2,3\}$,

$$
s_{j}\left(k_{j}\right)=\frac{1}{K_{j}}\left(1-\frac{\left|k_{j}\right|}{K_{j}}\right) \mathbb{1}_{\left[-K_{j}, K_{j}\right]}\left(k_{j}\right) .
$$

Consequently, we have supp $s_{j}=\left[-K_{j}, K_{j}\right], s_{j}\left(-k_{j}\right)=s_{j}\left(k_{j}\right)$ (yielding $s(-\mathbf{k})=s(\mathbf{k})$ and the quadrant symmetry), and the normalization $\int_{\left[-K_{j}, K_{j}\right]} s_{j}\left(k_{j}\right) d k_{j}=1$.

Correlation function and spatial correlation length without spectral measure uncertainties. From the spectrum separation, it can be deduced that, for all $\zeta=\left(\zeta_{1}, \zeta_{2}, \zeta_{3}\right)$ in $\mathbb{R}^{3}$, the correlation function is written as $\rho_{G}(\zeta)=\prod_{j=1}^{3} \rho_{j}\left(\zeta_{j}\right)$ in which, for $j \in\{1,2,3\}$,

$$
\begin{aligned}
& \rho_{j}\left(\zeta_{j}\right)=\int_{\mathbb{R}} e^{\imath k_{j} \zeta_{j}} s_{j}\left(k_{j}\right) d k_{j} \quad, \quad \rho_{j}(0)=1, \\
& L_{c j}=\int_{0}^{+\infty}\left|\rho_{j}\left(\zeta_{j}\right)\right| d \zeta_{j}=\pi s_{j}(0)=\pi / K_{j} .
\end{aligned}
$$

Without spectral measure uncertainties, this equation shows that the spatial correlation lengths are effectively driven by the support of the spectral measure.

Dimensionless spectral density function and spectral sampling. For all $\kappa_{=}\left(\kappa_{1}, \kappa_{2}, \kappa_{3}\right)$ in $\mathbb{R}^{3}$, the dimensionless spectral density function $\chi$ defined by Eq. (16) can be written as $\chi(\kappa)=\prod_{j=1}^{3} \chi_{j}\left(\kappa_{j}\right)$ such that, for $j \in\{1,2,3\}$,

$$
\chi_{j}\left(\kappa_{j}\right)=\left(1-\left|\kappa_{j}\right|\right) \mathbb{1}_{[-1,1]}\left(\kappa_{j}\right),
$$

and therefore, we effectively have (see Section 4.4.4), supp $\chi_{j}=[-1,1], \chi_{j}\left(-\kappa_{j}\right)=\chi_{j}\left(\kappa_{j}\right)$, and $\int_{\mathbb{R}} \chi_{j}\left(\kappa_{j}\right) d \kappa_{j}=1$. For all $\beta=\left(\beta_{1}, \beta_{2}, \beta_{3}\right) \in \mathcal{B}$,

$$
\chi_{\beta}^{\Delta}=\prod_{j=1}^{3} \chi_{j \beta_{j}}^{\Delta} \quad, \quad \chi_{j \beta_{j}}^{\Delta}=\left(2 / v_{s}\right) \chi_{j}\left(\kappa_{\beta_{j}}\right) .
$$

Definition of $q_{\widehat{\boldsymbol{\beta}}}\left([y] ; \delta_{s}\right)$ and construction of $a_{\boldsymbol{\beta}}([y])$. For all $\widehat{\boldsymbol{\beta}}=\left(\widehat{\beta}_{1}, \widehat{\beta}_{2}, \widehat{\beta}_{3}\right) \in \widehat{\mathcal{B}}$ with $\widehat{\beta}_{j} \in$ $\left\{1, \ldots, \widehat{v}_{s}\right\}$ and $j \in\{1,2,3\}, q_{\widehat{\beta}}\left([y] ; \delta_{s}\right)$ is defined by

$$
q_{\widehat{\beta}}\left([y] ; \delta_{s}\right)=\prod_{j=1}^{3} q_{\widehat{j} \widehat{\beta}_{j}}\left([y] ; \delta_{j}\right) \quad, \quad \forall[y] \in C_{y},
$$

in which for $j \in\{1,2,3\}$ and $\widehat{\beta}_{j} \in\left\{1, \ldots, \widehat{v}_{s}\right\}$,

$$
q_{\widehat{j} \widehat{\beta}_{j}}\left([y] ; \delta_{j}\right)=1+\underset{15}{\sqrt{12}} \delta_{j}\left([y]_{\widehat{j} \widehat{\beta}_{j}}-1 / 2\right),
$$


with $\delta_{j}>0$ the hyperparameter. We thus have

$$
a_{\widehat{\boldsymbol{\beta}}}([y])=\prod_{j=1}^{3} a_{\widehat{j}_{j}}([y]) \quad, \quad \widehat{\beta}_{j} \in\left\{1, \ldots, \widehat{v}_{s}\right\}
$$

in which

$$
\begin{aligned}
& a_{\widehat{\beta}_{j}}([y])=\sqrt{\chi_{j \widehat{\beta}_{j}}^{\Delta}} q_{\widehat{j}_{j}}\left([y], \delta_{j}\right), \widehat{\beta}_{j} \in\left\{1, \ldots, \widehat{v}_{s}\right\}, \\
& \left.a_{j \beta_{j}}([y])=a_{j,\left(2 \widehat{v}_{s}+1-\beta_{j}\right)}([y]), \beta_{j} \in \widehat{v}_{s}+1, \ldots, 2 \widehat{v}_{s}\right\} .
\end{aligned}
$$

Random variable $A_{\widehat{\beta}}$ and hyperparameter $\delta_{s}$. For $j \in\{1,2,3\}$ and $\widehat{\beta}_{j} \in\left\{1, \ldots, \widehat{v}_{s}\right\}$, the mean value and the second-order moment of random variable $A_{\overline{j \beta_{j}}}=a_{\widehat{j \beta_{j}}}([\mathbf{Y}])$ are

$$
E\left\{A_{\widehat{j}_{j}}\right\}=\sqrt{\chi_{\bar{j} \widehat{\beta}_{j}}^{\Delta}} \quad, \quad E\left\{A_{\widetilde{j}_{j}}^{2}\right\}=\chi_{\tilde{j} \widehat{\beta}_{j}}^{\Delta}\left(1+\delta_{j}^{2}\right) .
$$

Since the random variables $\left\{A_{\widehat{\beta}_{j}}\right\}_{j, \widehat{\beta}_{j}}$ are independent, the mean value and the second-order moment of the random variable $A_{\widehat{\beta}}=a_{\widehat{\beta}}([\mathbf{Y}])=\prod_{j=1}^{3} A_{\widehat{j}_{j}}$ are

$$
E\left\{A_{\vec{\beta}}\right\}=\sqrt{\chi_{\widehat{\beta}}^{\Delta}} \quad, \quad E\left\{A_{\widehat{\beta}}^{2}\right\}=\chi_{\widehat{\beta}}^{\Delta} \prod_{j=1}^{3}\left(1+\delta_{j}^{2}\right) .
$$

Defining the hyperparameter $\delta_{s}$ as

$$
\delta_{s}^{2}=E\left\{\left(A_{\widehat{\boldsymbol{\beta}}}-\sqrt{\chi_{\widehat{\boldsymbol{\beta}}}^{\Delta}}\right)^{2}\right\} / \chi_{\widehat{\boldsymbol{\beta}}}^{\Delta},
$$

it can be seen that we have $\delta_{s}^{2}=\left(\prod_{j=1}^{3}\left(1+\delta_{j}^{2}\right)\right)-1>0$, which is independent of $\widehat{\boldsymbol{\beta}}$.

Discretized dimensionless spectral measure. Eq. (20) yields

$$
\widetilde{\chi}_{\boldsymbol{\beta}}^{\Delta}([y])=\prod_{j=1}^{3} \widetilde{\chi}_{j \beta_{j}}^{\Delta}([y]), \forall \boldsymbol{\beta}=\left(\beta_{1}, \beta_{2}, \beta_{3}\right) \in \mathcal{B},
$$

in which, for $j \in\{1,2,3\}$ and $\beta_{j} \in\left\{1, \ldots, v_{s}\right\}$,

$$
\widetilde{\chi}_{j \beta_{j}}^{\Delta}([y])=a_{j \beta_{j}}([y])^{2}\left(\sum_{\beta^{\prime} \in \mathcal{B}} a_{\beta^{\prime}}([y])^{2}\right)^{-1 / 3} .
$$

Random discretized dimensionless spectral density function and random correlation lengths of $G^{v}(\cdot ;[y])$. For $j$ fixed in $\{1,2,3\}$, the random discretized dimensionless spectral density function $\kappa \mapsto \widetilde{\chi}_{j}(\kappa ;[\mathbf{Y}])$ from $[-1,1]$ into $\mathbb{R}^{+}$can be written as

$$
\widetilde{\chi}_{j}(\kappa ;[\mathbf{Y}])=\frac{v_{s}}{2} \sum_{\beta_{j}=1}^{v_{s}} \widetilde{\chi}_{j \beta_{j}}^{\Delta}([\mathbf{Y}]) \mathbb{1}_{\left[\kappa_{\beta_{j}}-\frac{1}{v_{s}}, \kappa_{\beta_{j}}+\frac{1}{\left.v_{s}\right]}\right]}(\kappa),
$$

in which $\widetilde{\chi}_{j \beta_{j}}^{\Delta}$ ([YY]) is given by Eq. (28) and where $\kappa_{\beta_{j}}$ is defined by Eq. (17). It can be seen that we effectively have $\int_{\mathbb{R}} \widetilde{\chi}_{j}(\kappa ;[\mathbf{Y}]) d \kappa=1$ a.s. 
Taking into account the change of variable $\zeta_{j}=\xi_{j} / K_{j}$ for $j \in\{1,2,3\}$ and Eq. (29), the random spatial correlation length of random field $G^{v}(\cdot ;[y])$ for coordinate $j$ is written as

$$
L_{c j}^{v}([y])=\frac{\pi}{K_{j}} \frac{v_{s}}{2} \widetilde{\chi}_{j \beta_{j}}^{\Delta}([y]) \quad \text { with } \quad \beta_{j}=v_{s} / 2 .
$$

The random spatial correlation length $\widetilde{L}_{c j}^{v}$ in presence of spectral measure uncertainties is thus given by

$$
\widetilde{L}_{c j}^{v}=L_{c j}^{v}([\mathbf{Y}]) .
$$

\subsection{Mean model at mesoscale of the microstructure}

Domain $\Omega$ is the cube $] 0,1\left[{ }^{3}\right.$ and the mean model of the elastic material at mesoscale is chosen in the isotropic class with mean Young modulus $\underline{Y}=10^{10}$ and mean Poisson coefficient $\underline{v}=0.15$ (the International System of Units is used).

\subsection{Random apparent elasticity field}

The level of statistical fluctuations of the random apparent elasticity field is controlled by hyperparameter $\delta_{c}$ defined by Eq. (7). For minimizing the number of computational cases, we will only consider two cases. One that corresponds to relatively small statistical fluctuations of the apparent elasticity field for which $\delta_{c}=0.2$ and another one for large statistical fluctuations for which $\delta_{c}=0.5$.

\subsection{Uncertain spectral measure}

The uncertainties in the spectral measure are controlled by the parameters $L_{c 1}, L_{c 2}, L_{c 3}$ (corresponding to the spatial correlations lengths when there are no uncertainties and controlling the support of the spectral measure with and without uncertainties) and by the spectrum dispersions $\delta_{1}, \delta_{2}, \delta_{3}$ inducing a global spectrum dispersion $\delta_{s}$ such that $\delta_{s}^{2}=\prod_{j=1}^{3}\left(1+\delta_{j}^{2}\right)-1$. For the computational stochastic homogenization analysis presented in this paper, it is assumed that $L_{c 1}=L_{c 2}=L_{c 3}=L_{c}$ with $L_{c} \in\{0.1,0.2,0.4,0.6\}$ and that $\delta_{1}=\delta_{2}=\delta_{3}=\delta$ with $\delta \in\{0.0,0.2,0.5\}$, which yields $\delta_{s}=\left(\left(1+\delta^{2}\right)^{3}-1\right)^{1 / 2} \in\{0.0,0.35,0.98\}$.

\subsection{Finite element discretization}

The weak formulation of the stochastic BVP is discretized by the finite element method. The finite element mesh of domain $\bar{\Omega}=[0,1]^{3}$ is made up of $n_{\mathrm{fem}} \times n_{\mathrm{fem}} \times n_{\mathrm{fem}}=n_{\mathrm{fem}}^{3}$ solid finite elements (8-nodes solid), $\left(n_{\mathrm{fem}}+1\right)^{3}$ nodes, and $3 \times\left(n_{\mathrm{fem}}+1\right)^{3}$ degrees of freedom (dof). There are $2^{3}$ integrations points in each finite element, which yields $8 \times n_{\mathrm{fem}}^{3}$ integrations points for the spatial discretization of the $\mathbb{M}_{6}^{+}$-valued random apparent elasticity field and consequently, yields $21 \times 8 \times n_{\mathrm{fem}}^{3}$ random terms. The optimal value of $n_{\mathrm{fem}}$ is estimated by the convergence analysis presented in Section 7.3. 


\section{Estimation of the parameters by using a convergence analysis}

\subsection{Definition of the convergence-analysis function}

Before performing the computational analysis of stochastic homogenization, we have to identify the optimal value of the parameters $n_{\mathrm{fem}}, v_{s}$, and $N$ that control the number $n_{\mathrm{fem}}^{3}$ of finite elements in the 3D-mesh, the spectral domain sampling, and the number of realizations used by the Monte Carlo numerical method. For all random matrix $[\mathbf{A}]$ defined on $(\Theta, \mathcal{T}, \mathcal{P})$ with values in $\mathbb{M}_{6}^{S}$, we define the norm $\|[\mathbf{A}]\|_{L^{2}}$ of $L_{\mathcal{P}}^{2}\left(\Theta, \mathbb{M}_{6}^{S}\right)$ such that its square is

$$
\|[\mathbf{A}]\|_{L^{2}}^{2}=E\left\{\|[\mathbf{A}]\|_{2}^{2}\right\}=\int_{\Theta}\|[\mathbf{A}(\theta)]\|_{2}^{2} d \mathcal{P}(\theta) .
$$

We introduce the convergence-analysis function

$$
\left(n_{\mathrm{fem}}, v_{s}, N\right) \mapsto \frac{\left\|\left[\widetilde{\mathbb{C}}^{\mathrm{eff}}\left(n_{\mathrm{fem}}, v_{s}, N\right)\right]\right\|_{L^{2}}^{2}}{\|[\underline{\mathbb{C}}]\|_{2}^{2}},
$$

which corresponds to the square of the $L^{2}$-norm of the $\mathbb{M}_{6}^{+}$-valued random effective elasticity matrix $\left[\widetilde{\mathbb{C}}^{\text {eff }}\left(n_{\mathrm{fem}}, v_{s}, N\right)\right]$ normalized with respect to the square $\|[\underline{\mathbb{C}}]\|_{2}^{2}$ of the norm of matrix $[\underline{\mathbb{C}}]=$ $E\{[\mathbb{C}(\mathbf{x},[y])]\} \in \mathbb{M}_{6}^{+}$that is independent of $\mathbf{x}$ and $[y]$. Let $\widetilde{\Lambda}_{1}\left(n_{\mathrm{fem}}, v_{s}, N\right) \geq \ldots \geq \widetilde{\Lambda}_{6}\left(n_{\mathrm{fem}}, v_{s}, N\right)>$ 0 be the ordered (a.s) random eigenvalues of $\left[\widetilde{\mathbb{C}}^{\text {eff }}\left(n_{\mathrm{fem}}, v_{s}, N\right)\right]$ and let $\underline{\lambda}_{1} \geq \ldots \geq \underline{\lambda}_{6}>0$ be the ordered eigenvalues of $[\underline{\mathbb{C}}]$. The convergence-analysis function defined by Eq. (33) can then be rewritten as

$$
\left(n_{\mathrm{fem}}, v_{s}, N\right) \mapsto E\left\{\Lambda_{1}\left(n_{\mathrm{fem}}, v_{s}, N\right)^{2}\right\},
$$

in which $\Lambda_{1}\left(n_{\mathrm{fem}}, v_{s}, N\right)$ is the normalization of the largest (a.s) random eigenvalue $\widetilde{\Lambda}_{1}\left(n_{\mathrm{fem}}, v_{s}, N\right)$,

$$
\Lambda_{1}\left(n_{\mathrm{fem}}, v_{s}, N\right)=\frac{\widetilde{\Lambda}_{1}\left(n_{\mathrm{fem}}, v_{s}, N\right)}{\underline{\lambda}_{1}}>0 \quad \text { a.s. }
$$

The construction of $\left(n_{\mathrm{fem}}, v_{s}, N\right) \mapsto E\left\{\Lambda_{1}\left(n_{\mathrm{fem}}, v_{s}, N\right)^{2}\right\}$ on a grid of $\mathbb{N}^{3}$ is too numerically expensive as soon as the three integers $n_{\mathrm{fem}}, v_{s}$, and $N$ simultaneously become large. We have therefore restricted the convergence analysis by constructing partial mappings.

\subsection{Convergence analysis with respect to the spectral domain sampling}

For performing the convergence with respect to the spectral domain sampling defined by the value of $v_{s}$ (the number of sampling points in the spectral domain is then $v=v_{s}^{3}$ ), we want to choose a value of $L_{c}$ that allows for obtaining significant statistical fluctuations for the random effective elasticity matrix $\left[\mathbb{C}^{\text {eff }}\right]$ (no scale separation) to check the mean-square convergence. Consequently we have taken the value $L_{c}=0.4$ that allows for choosing a moderate value $n_{\text {fem }}=$ 20 yielding 8000 finite elements (8-nodes solid elements) and 64000 integration points (this choice corresponds to 16 integration points by spatial correlation length $L_{c j}=L_{c}$ for each space coordinate $j$, which yields a good numerical approximation). In addition, in order to take into account the larger statistical fluctuations (worst case for convergence), we have taken $\delta=0.5$ and $\delta_{c}=0.5$. Figure 2 displays the graph of function $v_{s} \mapsto E\left\{\Lambda_{1}\left(v_{s}\right)^{2}\right\}=E\left\{\Lambda_{1}\left(n_{\mathrm{fem}}, v_{s}, N\right)^{2}\right\}$ for $n_{\mathrm{fem}}=20, N=1000$, and for $v_{s} \in[2,30]$ that is to say for $v \in[8,27000]$. It can be seen that $v_{s}=18$, yielding $v=5832$, is a good compromise. For this value of $v_{s}$, Eq. (15) holds (we even have strict equality at 1 ). 


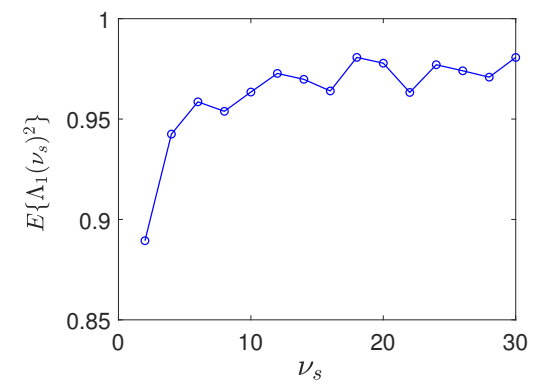

Figure 2: Convergence with respect to the spectral domain sampling: graph of function $v_{s} \mapsto E\left\{\Lambda_{1}\left(v_{s}\right)^{2}\right\}$.

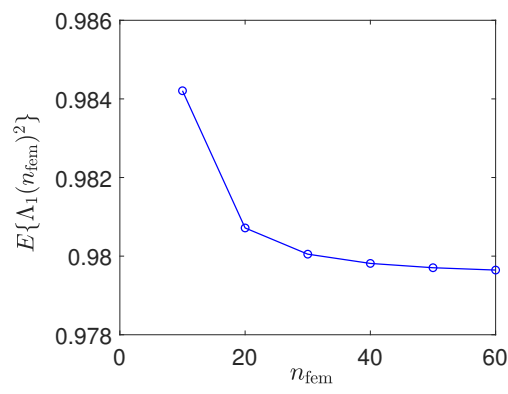

Figure 3: Convergence with respect to the mesh: graph of function $n_{\mathrm{fem}} \mapsto E\left\{\Lambda_{1}\left(n_{\mathrm{fem}}\right)^{2}\right\}$.

\subsection{Convergence analysis with respect to the mesh}

The worst case for the convergence analysis with respect to the number $n_{\mathrm{fem}}^{3}$ of finite elements corresponds to the smallest considered value of $L c$ and to the largest value for $\delta$ and for $\delta_{c}$, yielding $L_{c}=0.1, \delta=0.5$, and $\delta_{c}=0.5$. Figure 3 displays the graph of function $n_{\mathrm{fem}} \mapsto$ $E\left\{\Lambda_{1}\left(n_{\mathrm{fem}}\right)^{2}\right\}=E\left\{\Lambda_{1}\left(n_{\mathrm{fem}}, v_{s}, N\right)^{2}\right\}$ for $v_{s}=18, N=1000$, and for $n_{\mathrm{fem}} \in[10,60]$ that is to say for $n_{\mathrm{fem}}^{3} \in[1000,216000]$. It can be seen that $n_{\mathrm{fem}}=50$ is a good value (yielding $n_{\mathrm{fem}}^{3}=125000$ finite elements and 1000000 integration points).

\subsection{Convergence analysis with respect to the number of realizations}

The worst case for the convergence analysis with respect to the number $N$ of realizations corresponds to the smallest considered value of $L_{c}$ and to the largest value for $\delta$ and for $\delta_{c}$, yielding $L_{c}=0.1, \delta=0.5$, and $\delta_{c}=0.5$. Figure 4 displays the graph of function $N \mapsto E\left\{\Lambda_{1}(N)^{2}\right\}=$ $E\left\{\Lambda_{1}\left(n_{\text {fem }}, v_{s}, N\right)^{2}\right\}$ for $n_{\text {fem }}=50, v_{s}=18$, and for $N \in[1,1000]$. It can be seen that $N=1000$ is a satisfactory value.

\subsection{Example of realizations of the random discretized dimensionless spectral measure}

For the considered separable spatial correlation structure (see Section 6.1) and for coordinate $j$ fixed in $\{1,2,3\}$, the random discretized dimensionless spectral density function $\kappa \mapsto \widetilde{\chi}_{j}(\kappa ;[\mathbf{Y}])$ from $[-1,1]$ into $\mathbb{R}^{+}$is given by Eq. (29). By way of illustration, Figs. 5, 6, and 7 shows, for $j=1$ and for $v_{s}=18$, a realization $\kappa \mapsto \widetilde{\chi}_{j}\left(\kappa ;\left[y^{\ell}\right]\right)$ for $\delta=0$ (no uncertainties and thus independent of realization $\left[y^{\ell}\right]$ of $\left.[\mathbf{Y}]\right)$, and for $\delta=0.2$ and 0.5 for a realization $\left[y^{\ell}\right]$ of $[\mathbf{Y}]$. 


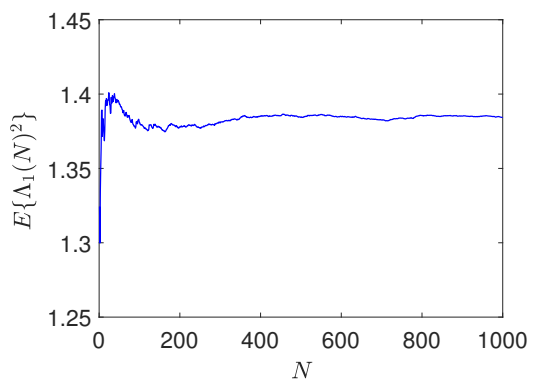

Figure 4: Convergence with respect to the number of realizations: graph of function $N \mapsto E\left\{\Lambda_{1}(N)^{2}\right\}$.

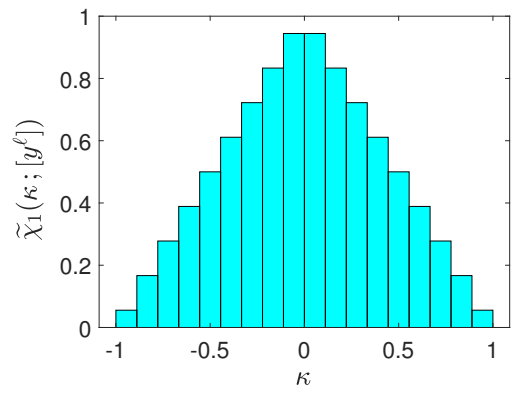

Figure 5: For $\delta=0.0$ (no uncertainties), graph of $\kappa \mapsto \widetilde{\chi}_{1}\left(\kappa ;\left[y^{\ell}\right]\right)$ (independent of realization $\left[y^{\ell}\right]$ of $[\mathbf{Y}]$.

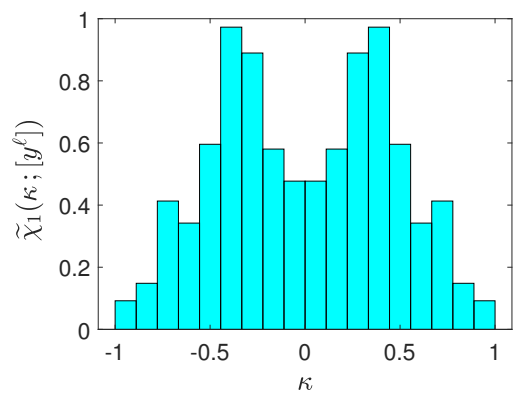

Figure 6: For $\delta=0.2$, graph of realization $\kappa \mapsto \widetilde{\chi}_{j}\left(\kappa ;\left[y^{\ell}\right]\right)$ for a realization $\left[y^{\ell}\right]$ of $[\mathbf{Y}]$. 


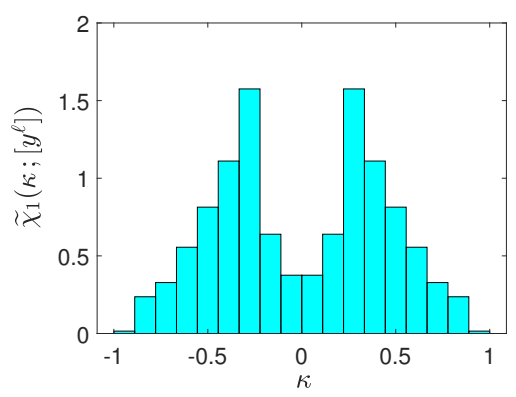

Figure 7: For $\delta=0.5$, graph of realization $\kappa \mapsto \widetilde{\chi}_{j}\left(\kappa ;\left[y^{\ell}\right]\right)$ for a realization $\left[y^{\ell}\right]$ of $[\mathbf{Y}]$.

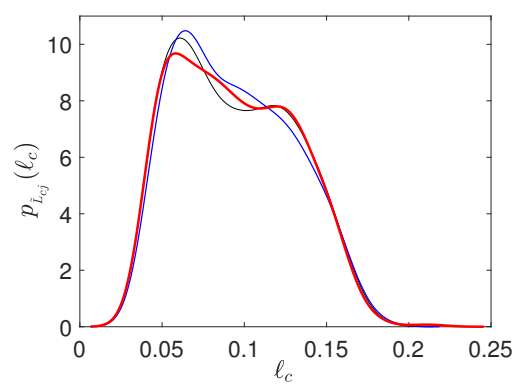

Figure 8: For $L_{c}=0.1$ and $\delta=0.2$, graph of pdf $\ell_{c} \mapsto p_{\tilde{L}_{c j}^{v}}\left(\ell_{c}\right)$ of random variable $\widetilde{L}_{c j}^{v}$ for $j=1$ (thin black), $j=2$ (med blue), and $j=3$ (thick red).

\subsection{Probabilistic analysis of the random correlation lengths of random field $G^{v}(.,[Y])$}

For $j \in\{1,2,3\}$, we propose a probabilistic analysis of the random correlation length $\widetilde{L}_{c j}^{v}$ defined by Eq. (31) with Eq. (30). It should be noted that random variable $\widetilde{L}_{c j}^{v}$ depends on $\delta$ (controlling the level of uncertainties in the spectral measure) and on $\pi / K_{j}=L_{c j}$ (see Eq. (26)) for which we have chosen $L_{c j}=L_{c}$. Let $m_{\tilde{L}_{c j}^{v}}=E\left\{\widetilde{L}_{c j}^{v}\right\}$ be the mean value of $\widetilde{L}_{c j}^{v}$ and $\delta_{\tilde{L}_{c j}^{v}}$ its coefficient of variation, which are estimated using $N_{d}=1000$.

- Mean value $m_{\tilde{L}_{c j}^{v}}$ does not really depends on $\delta$ (for $\delta=0.0,0.2$, and 0.5 ) and we have $m_{\tilde{L}_{c 1}^{v}} \simeq m_{\tilde{L}_{c 2}^{v}} \simeq m_{\tilde{L}_{c 3}^{v}} \simeq m_{\tilde{L}_{c}^{v}}$ (that was expected). For $L_{c}=0.1,0.2,0.4$, and 0.6 , the estimation of $m_{\tilde{L}_{c}^{v}}$ is $0.096,0.19,0.38$, and 0.57 .

- Coefficient of variation $\delta_{\tilde{L}_{c j}^{v}}$ does not depends on $L_{c}$ (that can easily be seen) and we have $\delta_{\tilde{L}_{c 1}^{v}} \simeq \delta_{\tilde{L}_{c 2}^{v}} \simeq \delta_{\tilde{L}_{c 3}^{v}} \simeq \delta_{\tilde{L}_{c}^{v}}$ (that was expected). For $\delta=0.0,0.2$, and 0.5, the estimation of $\delta_{\tilde{L}_{c}^{v}}$ is $0.0,0.37$, and 0.82 .

As an illustration, for $L_{c}=0.1$ and $\delta=0.2$, Fig. 8 displays the graph of the probability density function $\ell_{c} \mapsto p_{\tilde{L}_{c j}^{v}}\left(\ell_{c}\right)$ of random variable $\widetilde{L}_{c j}^{v}$ for $j=1,2,3$. Note that these three probability density functions would exactly be the same for $N \rightarrow+\infty$. 


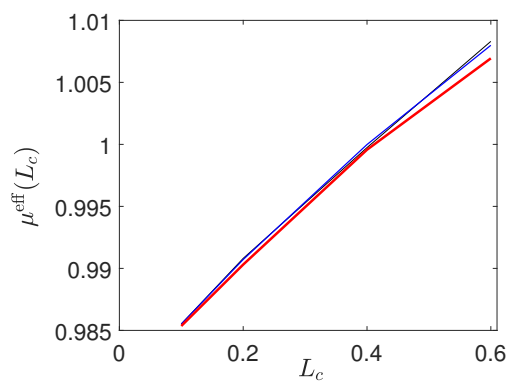

Figure 9: For $\delta_{c}=0.2$, graph of $L_{c} \mapsto \mu^{\text {eff }}\left(L_{c}\right)$ as a function of the level $\delta=0.0$ (thin black), 0.2 (med blue), and 0.5 (thick red) of spectral measure uncertainties.

\section{Computational stochastic homogenization analysis}

Taking into account the convergence analysis presented in Section 7, the computational stochastic homogenization analysis is performed with $n_{\mathrm{fem}}=50, v_{s}=18$, and $N=1000$. The sensitivity of the probabilistic analysis of the RVE size with respect to the uncertainty level of the spectral measure is performed by analyzing the normalized random variable $Z^{\text {eff }}$ defined by

$$
Z^{\mathrm{eff}}=\frac{\left\|\left[\widetilde{\mathbb{C}}^{\text {eff }}\right]\right\|_{2}}{E\left\{\left\|\left[\widetilde{\mathbb{C}}^{\text {eff }}\right]\right\|_{2}\right\}}=\frac{\widetilde{\Lambda}_{1}}{E\left\{\widetilde{\Lambda}_{1}\right\}} .
$$

Let $\mu^{\text {eff }}$ be defined by

$$
\mu^{\mathrm{eff}}=\frac{E\left\{\left\|\left[\widetilde{\mathbb{C}}^{\mathrm{eff}}\right]\right\|_{2}\right\}}{\|[\underline{\mathbb{C}}]\|_{2}}=\frac{E\left\{\widetilde{\Lambda}_{1}\right\}}{\underline{\lambda}_{1}} .
$$

From Eqs. (35) to (37), it can be seen that $\Lambda_{1}=\mu^{\text {eff }} Z^{\text {eff. }}$. The normalized mean value $\mu^{\text {eff }}$ depends on $\delta_{c}, L_{c}$, and $\delta$. For the two values $\delta_{c}=0.2$ and 0.5 that control the statistical fluctuations of the random apparent elasticity field, Figs. 9 and 10 show the graph of function $L_{c} \mapsto \mu^{\text {eff }}\left(L_{c}\right)$ (defined by Eq. (37)) as a function of the values of $\delta=0.0,0.2$, and 0.5 that define the level of spectral measure uncertainties (there are no uncertainties for $\delta=0.0$ ). Let $\eta \mapsto P(\eta)$ be the function from ] $0,1]$ into $[0,1]$ defined by

$$
\begin{aligned}
P(\eta) & =\operatorname{Proba}\left\{1-\eta<Z^{\mathrm{eff}} \leq 1+\eta\right\} \\
& =F_{Z^{\mathrm{eff}}}(1+\eta)-F_{Z^{\mathrm{eff}}}(1-\eta),
\end{aligned}
$$

in which $F_{Z^{\text {eff }}}$ is the cumulative distribution function of $Z^{\text {eff }}$ that is estimated with the Gaussian kernel cumulative distribution estimation method [66] and with the $N$ independent realizations of $Z^{\text {eff }}$.

\subsection{Probabilistic analysis of the RVE size without uncertainties in the spectral measure}

For $\delta=0$ (no uncertainties in the spectral measure) and for statistical fluctuations of the random apparent elasticity field corresponding to the two values $\delta_{c}=0.2$ and 0.5 , Figs. 11 and 12 show the graph of function $\eta \mapsto P(\eta)$ (defined by Eq. (38)) as a function of $L_{c}=0.1,0.2,0.4$, and 0.6. 


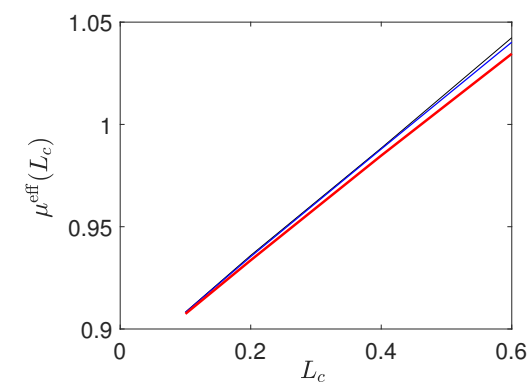

Figure 10: For $\delta_{c}=0.5$, graph of $L_{c} \mapsto \mu^{\text {eff }}\left(L_{c}\right)$ as a function of the level $\delta=0.0$ (thin black), 0.2 (med blue), and 0.5 (thick red) of spectral measure uncertainties.

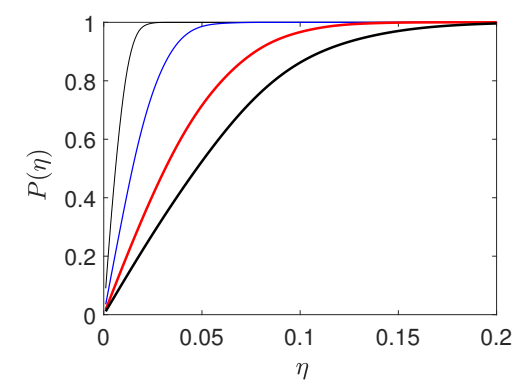

Figure 11: For $\delta_{c}=0.2$ and for $\delta=0$ (no uncertainty), graph of $\eta \mapsto P(\eta)$ for $L_{c}=0.1$ (thin black), 0.2 (med blue ), 0.4 (thick red), and 0.6 (thick black).

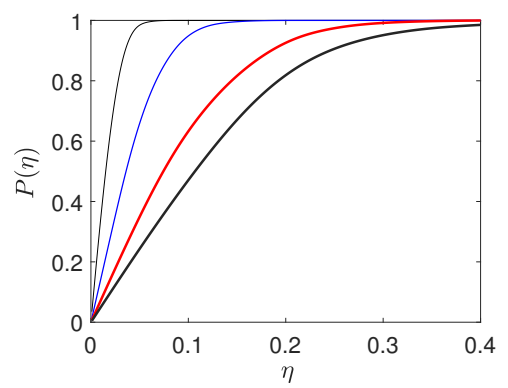

Figure 12: For $\delta_{c}=0.5$ and for $\delta=0$ (no uncertainty), graph of $\eta \mapsto P(\eta)$ for $L_{c}=0.1$ (thin black), 0.2 (med blue), 0.4 (thick red), and 0.6 (thick black). 


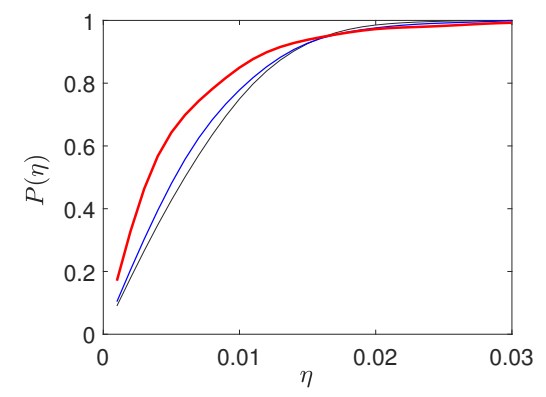

Figure 13: For $L_{c}=0.1$ and $\delta_{c}=0.2$, graph of $\eta \mapsto P(\eta)$ for $\delta=0$ (thin black), 0.2 (med blue), 0.5 (thick red).

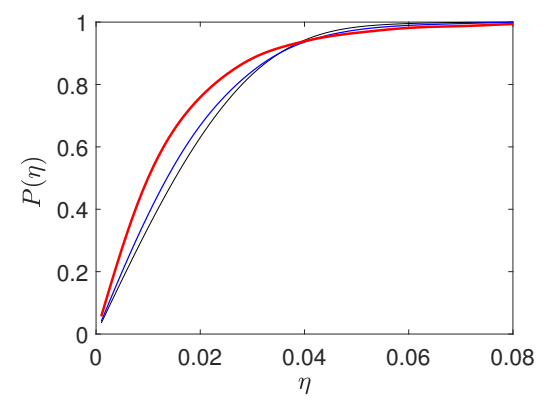

Figure 14: For $L_{c}=0.1$ and $\delta_{c}=0.5$, graph of $\eta \mapsto P(\eta)$ for $\delta=0$ (thin black), 0.2 (med blue), 0.5 (thick red).

\subsection{Probabilistic analysis of the RVE size with uncertainties in the spectral measure}

For the two values $\delta_{c}=0.2$ and 0.5 controlling the level of statistical fluctuations of the random apparent elasticity field and for $L_{c}=0.1,0.2,0.4,0.6$, Figs. 13 to 20 show the graph of function $\eta \mapsto P(\eta)$ (defined by Eq. (38)) as a function of the level $\delta=0.0,0.2,0.5$ of uncertainties in the spectral measure (for $\delta=0$ there are no uncertainties).

\subsection{Synthesis of the results and discussion}

Analysis for the case $L_{c}=0.1$. For $L_{c}=0.1$, for $\delta_{c}=0.2$ (relatively small statistical fluctuations of the apparent elasticity field) and $\delta_{c}=0.5$ (large statistical fluctuations), Table 1 gives the value of the probability $P(\eta)=\operatorname{Proba}\left\{1-\eta<Z^{\text {eff }} \leq 1+\eta\right\}$ (see Eq. (38)) for $\eta=0.05,0.02$, 0.01 , and for $\delta=0$ (no uncertainties), $\delta=0.2$ and 0.5 (uncertainties in the spectral measure). The values given in Table 1 are extracted from Figs. 13 and 14. The value of the probability for that the random variable $Z^{\text {eff }}$ belongs to interval $\left.] 0.95,1.05\right]$ shows that we obtain a perfect scale separation for $\delta_{c}=0.2$ (probability is 1) and an excellent one for $\delta_{c}=0.5$ (probability larger than 0.966) for both the small and large uncertainties in the spectral measure. For large uncertainties $(\delta=0.5)$, it can also be seen that the value of the probability for that $Z^{\text {eff }}$ belongs to $] 0.98,1.02$ ] or $] 0.99,1.01$ ] decreases and becomes too small for validating a scale separation. Finally, we can also see (and this will also be true for the largest values of $L_{c}$ ) that the probability increases with $\delta$ (contrary to what was expected). This is because as $\delta$ increases, the dispersion of the random spatial correlation lengths increases favoring small values, which "helps" the scale separation (see Section 7.6 and in particular Fig. 8). In summary, the scale separation is achieved 


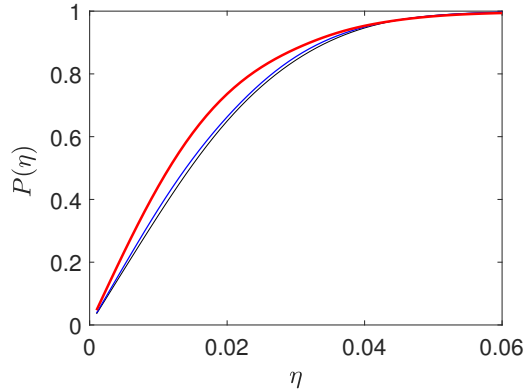

Figure 15: For $L_{c}=0.2$ and $\delta_{c}=0.2$, graph of $\eta \mapsto P(\eta)$ for $\delta=0$ (thin black), 0.2 (med blue), 0.5 (thick red).

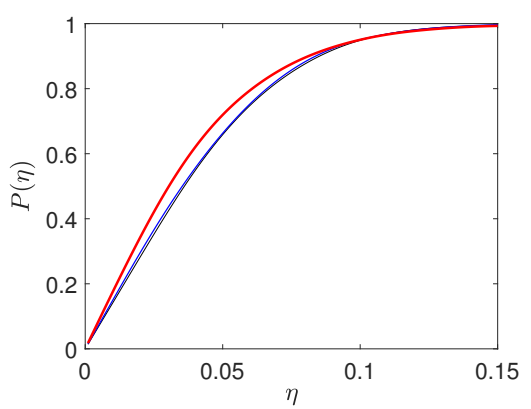

Figure 16: For $L_{c}=0.2$ and $\delta_{c}=0.5$, graph of $\eta \mapsto P(\eta)$ for $\delta=0$ (thin black), 0.2 (med blue), 0.5 (thick red).

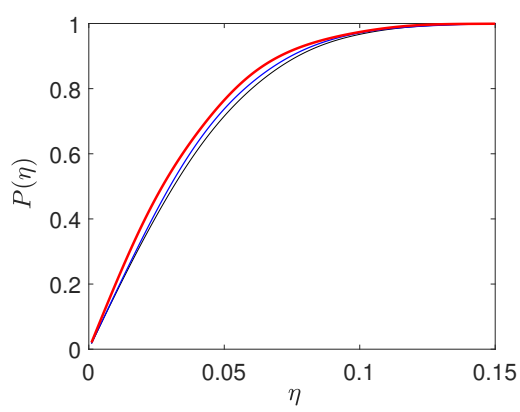

Figure 17: For $L_{c}=0.4$ and $\delta_{c}=0.2$, graph of $\eta \mapsto P(\eta)$ for $\delta=0$ (thin black), 0.2 (med blue), 0.5 (thick red). 


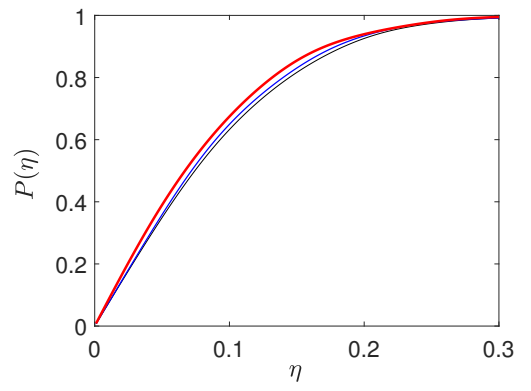

Figure 18: For $L_{c}=0.4$ and $\delta_{c}=0.5$, graph of $\eta \mapsto P(\eta)$ for $\delta=0$ (thin black), 0.2 (med blue), 0.5 (thick red).

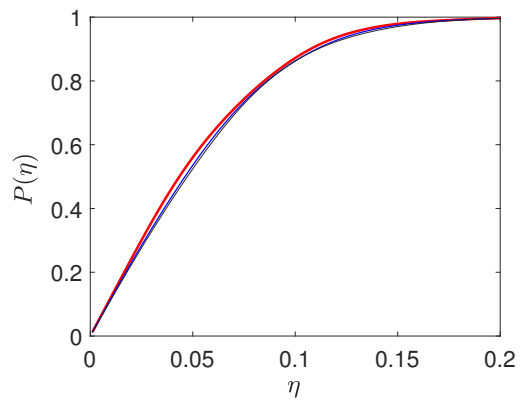

Figure 19: For $L_{c}=0.6$ and $\delta_{c}=0.2$, graph of $\eta \mapsto P(\eta)$ for $\delta=0$ (thin black), 0.2 (med blue), 0.5 (thick red).

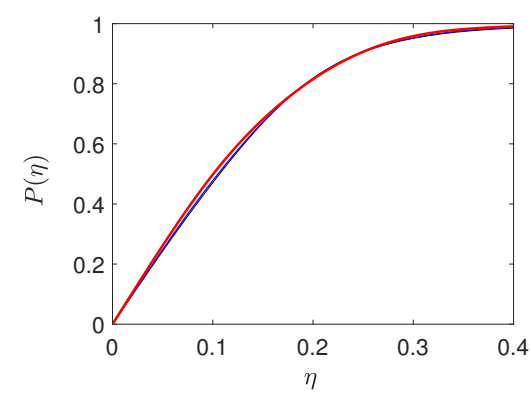

Figure 20: For $L_{c}=0.6$ and $\delta_{c}=0.5$, graph of $\eta \mapsto P(\eta)$ for $\delta=0$ (thin black), 0.2 (med blue), 0.5 (thick red). 
at 5 percent, for both small and large uncertainties in the spectral measure and the uncertainties in the spectral measure favor the separation of scale.

Table 1: For $L_{c}=0.1$ and for statistical fluctuations of the random apparent elasticity field corresponding to $\delta_{c}=0.2$ and $\delta_{c}=0.5$, sensitivity of the probabilistic analysis of the RVE size with respect to the uncertainty level $\delta$ in the spectral measure.

\begin{tabular}{|c|c|c|c|c|}
\hline$\delta_{c}$ & $\delta$ & $\operatorname{Proba}\left\{Z^{\text {eff }} \in\right.$ & Proba $\left\{Z^{\text {eff }} \in\right.$ & Proba $\left\{Z^{\text {eff }} \in\right.$ \\
$10.95,1.05]\}$ & ] $0.98,1.02]\}$ & ] $0.99,1.01]\}$ \\
\hline 0.2 & 0.0 & 1.0 & 0.985 & 0.750 \\
& 0.2 & 1.0 & 0.976 & 0.778 \\
& 0.5 & 1.0 & 0.973 & 0.849 \\
\hline 0.5 & 0.0 & 0.984 & 0.630 & 0.341 \\
& 0.2 & 0.975 & 0.669 & 0.383 \\
& 0.5 & 0.966 & 0.759 & 0.500 \\
\hline
\end{tabular}

Analysis for the case $L_{c} \geq 0.2$. For $\delta_{c}=0.2,0.5$ and for $L_{c}=0.2,0.4,0.6$, Table 2 gives the value of the probability $\left.\left.\operatorname{Proba}\left\{Z^{\text {eff }} \in\right] 0.95,1.05\right]\right\}$ (see Eq. (38)) for $\delta=0$ (no uncertainties), $\delta=0.2$ and 0.5 (small and large uncertainties in the spectral measure). The values given in Table 2 are extracted from Figs. 15 and 16 for $L_{c}=0.2$, Figs. 17 and 18 for $L_{c}=0.4$, and Figs. 19 and 20 for $L_{c}=0.6$.

For $L_{c}=0.2$, the value of the probability for that the random variable belongs to interval ]0.95, 1.05]\} shows an excellent scale separation for $\delta_{c}=0.2$ but there is no scale separation for a large value of uncertainties $\left(\delta_{c}=0.5\right)$. For $L_{c}=0.4$ and $L_{c}=0.6$ there is no scale separation in all the cases (with no uncertainties $(\delta=0)$ or with small or large uncertainties $(\delta=0.2$ or 0.5$)$ ).

In summary, for these cases, the results obtained are consistent with the knowledge that we have concerning the probabilistic analysis of the RVE size [40]. For $L_{c}=0.2$, the scale separation is achieved at 5 percent for small uncertainties but is not obtained for larger uncertainties. For $L_{c}=0.4$ and $L_{c}=0.6$ the scale separation is never obtained for all values of $\delta$ (with no uncertainties and with uncertainties). Finally, as previously observed for the case $L_{c}=0.1$, the uncertainties in the spectral measure favor the separation of scale. However, this phenomenon is all the more important as $L_{c}$ is small (smaller than 0.2) and tends to attenuate when $L_{c}$ increases, almost vanishing for $L_{c}=0.6$.

CPU time for performing the presented analysis. The CPU time that has been required for performing the computational stochastic homogenization analysis is 32000 hours $\simeq 24$ (cases) $\times 44000$ (elapsed time) $\times 110$ (cores) $/ 3600$, in which the elapsed time is in seconds.

\section{Conclusion}

The uncertainties in the spectral density function of the random apparent elasticity field at mesoscale have a significant influence on the statistics of the random effective elasticity tensor at macroscale.

Although a more general probabilistic model of uncertainties in the spectral density function has been presented, the computational stochastic homogenization has been carried out using a 
Table 2: For $L_{c}=0.2,0.4,0.6$, and for statistical fluctuations of the random apparent elasticity field corresponding to $\delta_{c}=0.2$ and $\delta_{c}=0.5$, sensitivity of the probabilistic analysis of the RVE size with respect to the uncertainty level $\delta$ in the spectral measure.

\begin{tabular}{|c|c|c|c|}
\hline & & $\begin{array}{c}\operatorname{Proba}\left\{Z^{\mathrm{eff}} \in\right. \\
] 0.95,1.05]\}\end{array}$ & $\begin{array}{c}\operatorname{Proba}\left\{Z^{\mathrm{eff}} \in\right. \\
] 0.95,1.05]\}\end{array}$ \\
\hline$L_{c}$ & $\delta$ & $\delta_{c}=0.2$ & $\delta_{c}=0.5$ \\
\hline 0.2 & 0.0 & 0.986 & 0.658 \\
& 0.2 & 0.985 & 0.660 \\
& 0.5 & 0.983 & 0.719 \\
\hline 0.4 & 0.0 & 0.715 & 0.349 \\
& 0.2 & 0.738 & 0.356 \\
& 0.5 & 0.765 & 0.390 \\
\hline 0.6 & 0.0 & 0.524 & 0.243 \\
& 0.2 & 0.535 & 0.248 \\
& 0.5 & 0.560 & 0.262 \\
\hline
\end{tabular}

separable spatial correlation structure. In this model, the support of the spectral density function is parameterized by a length $L_{c}$ that appears as a deterministic spatial correlation length for each space coordinate (when there are no uncertainties). The uncertainties in the spectral density function are then taken into account by introducing a random shape of this function for which its support is given. These random shape implies random spatial correlation lengths for the random apparent elasticity field at mesoscale. Consequently, the proposed random field model with uncertainties on the spectral measure is richer in terms of modeling of random media than a simple parametric model of the spatial correlation lengths in the physical domain.

Let $L_{\Omega}$ be the dimension of the side of $\Omega$. The separation of scales is obtained when random effective elasticity tensor at macroscale exhibits negligible statistical fluctuations. For $L_{c} / L_{\Omega}=$ 0.1 , the scale separation is achieved at 5 percent, for both small and large uncertainties in the spectral density function of the apparent elasticity field; this means that domain $\Omega$ is then a RVE. Such a separation is lost for $L_{c} / L_{\Omega} \geq 0.2$ for all level of uncertainties (no uncertainties, small and large uncertainties). However, for $L_{c} / L_{\Omega}=0.2$ and for a small level of uncertainties $(\delta \leq 0.2)$, the scale separation is also achieved at 5 percent.

An unexpected results is that, for the "uniform" probabilistic model that has been proposed for the uncertainties in the spectral density function, these uncertainties favor the separation of scale in the computational stochastic homogenization. This phenomenon is all the more important as $L_{c} / L_{\Omega}$ is small (smaller than 0.2 ) and tends to attenuate when $L_{c} / L_{\Omega}$ increases, almost vanishing for $L_{c} / \Omega_{L}=0.6$.

We have presented this work within the framework of the following hypotheses: the mean value of the random apparent elasticity tensor at the mesoscopic scale has been taken in the class of isotropic media and the statistical fluctuations have been taken in the anisotropic class. The developed probabilistic modeling and the computational stochastic homogenization that have been carried out can be extended to more general situations using more complex material symmetry classes with mixture of symmetry classes for the statistical fluctuations of the apparent elasticity field. In particular, such extensions would be useful for analyzing the stochastic homogenization of thin layer that is homogenizable in the layer plane but not in the layer thickness and for which 
the three spatial correlation lengths and the level of uncertainties in the spectral density function are different as a function of the three space coordinates.

Finally, the parametric study with respect to $L_{c}, \delta_{c}$, and $\delta$ has been limited for the computational stochastic homogenization analysis because of the numerical costs that are relatively high. We could extend it while keeping numerical costs bearable by using probabilistic learning on manifolds or other machine learning approaches. However, in order to limit the length of the presentation and in order not to mix the issues presented in this paper, we have preferred to strictly limit the extent of the parametric study.

\section{Conflict of interest}

The author declares that they have no conflict of interest.

\section{References}

[1] E. Kröner, Statistical Continuum Mechanics, Springer-Verlag, 1971.

[2] E. Kröner, Bounds for effective elastic moduli of disordered materials, Journal of the Mechanics and Physics of Solids 25 (2) (1977) 137-155. doi:10.1016/0022-5096(77)90009-6.

[3] S. Nemat-Nasser, M. Hori, Micromechanics: Overall Properties of Heterogeneous Materials. Second revised edition, Elsevier, Amsterdam, 1999.

[4] G. W. Milton, The Theory of Composites, Cambridge University Press, Cambridge, UK, 2002 doi: $10.1017 /$ CBO9780511613357.

[5] S. Torquato, Random Heterogeneous Materials, Microstructure and Macroscopic Properties, Springer-Verlag, New York, 2002. doi:10.1007/978-1-4757-6355-3.

[6] E. Sánchez-Palencia, Non-Homogeneous Media and Vibration Theory, Vol. 127 of Lecture notes in physics, Springer-Verlag, Berlin, 1980.

[7] S. Forest, Homogenization methods and mechanics of generalized continua-part 2, Theoretical and applied mechanics 28-29 (2002) 113-143.

[8] A. Zaoui, Continuum micromechanics: survey, Journal of Engineering Mechanics 128 (8) (2002) 808-816. doi:10.1061/(ASCE)0733-9399(2002)128:8(808)

[9] A. Bourgeat, A. Piatnitski, Approximations of effective coefficients in stochastic homogenization, Annales de l'Institut Henri Poincare (B), Probability and Statistics 40 (2) (2004) 153-165. doi:10.1016/j.anihpb.2003.07.003.

[10] J. Yvonnet, Q.-C. He, C. Toulemonde, Numerical modelling of the effective conductivities of composites with arbitrarily shaped inclusions and highly conducting interface, Composites Science and Technology 68 (13) (2008) 2818-2825. doi:10.1016/j.compscitech.2008.06.008.

[11] P. Suquet, Continuum Micromechanics, Springer-Verlag, Wien, 2014.

[12] G. Papanicolaou, S. Varadhan, Boundary value problems with rapidly oscillating random coefficients, North Holland, 1981.

[13] S. Torquato, G. Stell, Microstructure of two-phase random media. v. the n-point matrix probability functions for impenetrable spheres, The Journal of chemical physics 82 (2) (1985) 980-987. doi:10.1063/1.448475.

[14] G. Nguetseng, A general convergence result for a functional related to the theory of homogenization, SIAM Journal on Mathematical Analysis 20 (3) (1989) 608-623. doi:10.1137/0520043.

[15] G. Allaire, Homogenization and two-scale convergence, SIAM Journal on Mathematical Analysis 23 (6) (1992) 1482-1518. doi:10.1137/0523084.

[16] K. Sab, On the homogenization and the simulation of random materials, European Journal of Mechanics, A/Solids 11 (5) (1992) 585-607.

[17] K. T. Andrews, S. Wright, Stochastic homogenization of elliptic boundary-value problems with Lp-data, Asymptotic Analysis 17 (3) (1998) 165-184.

[18] M. Ostoja-Starzewski, Random field models of heterogeneous materials, International Journal of Solids and Structures 35 (19) (1998) 2429-2455. doi:10.1016/S0020-7683(97)00144-3.

[19] V. V. Jikov, S. M. Kozlov, O. A. Oleinik, Homogenization of Differential Operators and Integral Functionals, Springer-Verlag, Berlin, Heidelberg, 2012. doi:10.1007/978-3-642-84659-5.

[20] W. K. Liu, C. McVeigh, Predictive multiscale theory for design of heterogeneous materials, Computational Mechanics 42 (2) (2008) 147-170. doi:10.1007/s00466-007-0176-8. 
[21] W. K. Liu, L. Siad, R. Tian, S. Lee, D. Lee, X. Yin, W. Chen, S. Chan, G. B. Olson, L.-E. Lindgen, et al., Complexity science of multiscale materials via stochastic computations, International Journal for Numerical Methods in Engineering 80 (6-7) (2009) 932-978. doi:10.1002/nme.2578

[22] W. K. Liu, D. Qian, S. Gonella, S. Li, W. Chen, S. Chirputkar, Multiscale methods for mechanical science of complex materials: Bridging from quantum to stochastic multiresolution continuum, International journal for numerical methods in engineering 83 (8-9) (2010) 1039-1080. doi:10.1002/nme.2915.

[23] J. Guilleminot, A. Noshadravanb, C. Soize, R. Ghanem, A probabilistic model for bounded elasticity tensor random fields with application to polycrystalline microstructures, Computer Methods in Applied Mechanics and Engineering 200 (17-20) (2011) 1637-1648. doi:10.1016/j.cma.2011.01.016.

[24] M. S. Greene, Y. Liu, W. Chen, W. K. Liu, Computational uncertainty analysis in multiresolution materials via stochastic constitutive theory, Computer Methods in Applied Mechanics and Engineering 200 (1-4) (2011) 309325. doi:10.1016/j.cma.2010.08.013.

[25] T.-T. Le, J. Guilleminot, C. Soize, Stochastic continuum modeling of random interphases from atomistic simulations. application to a polymer nanocomposite, Computer Methods in Applied Mechanics and Engineering 303 (2016) 430-449. doi:10.1016/j.cma.2015.10.006.

[26] C. Yu, O. L. Kafka, W. K. Liu, Multiresolution clustering analysis for efficient modeling of hierarchical material systems, Computational Mechanics (2021) 1-14doi:10.1007/s00466-021-01982-x.

[27] Z. Liu, J. A. Moore, S. M. Aldousari, H. S. Hedia, S. A. Asiri, W. K. Liu, A statistical descriptor based volumeintegral micromechanics model of heterogeneous material with arbitrary inclusion shape, Computational Mechanics 55 (5) (2015) 963-981. doi:10.1007/s00466-015-1145-2.

[28] M. A. Bessa, R. Bostanabad, Z. Liu, A. Hu, D. W. Apley, C. Brinson, W. Chen, W. K. Liu, A framework for data-driven analysis of materials under uncertainty: Countering the curse of dimensionality, Computer Methods in Applied Mechanics and Engineering 320 (2017) 633-667. doi:10.1016/j.cma.2017.03.037.

[29] C. Soize, Design optimization under uncertainties of a mesoscale implant in biological tissues using a probabilistic learning algorithm, Computational Mechanics 62 (3) (2018) 477-497. doi:10.1007/s00466-017-1509-X.

[30] H. Yang, X. Guo, S. Tang, W. K. Liu, Derivation of heterogeneous material laws via data-driven principal component expansions, Computational Mechanics 64 (2) (2019) 365-379. doi:10.1007/s00466-019-01728-w.

[31] R. Ghanem, C. Soize, L. Mehrez, V. Aitharaju, Probabilistic learning and updating of a digital twin for composite material systems, International Journal for Numerical Methods in Engineering online. doi:10.1002/nme.6430.

[32] C. Soize, R. Ghanem, C. Desceliers, Sampling of bayesian posteriors with a non-gaussian probabilistic learning on manifolds from a small dataset, Statistics and Computing 30 (5) (2020) 1433-1457. doi:10.1007/s11222-02009954-6.

[33] F. Pled, C. Desceliers, T. Zhang, A robust solution of a statistical inverse problem in multiscale computational mechanics using an artificial neural network, Computer Methods in Applied Mechanics and Engineering 373 (2021) 113540. doi:10.1016/j.cma.2020.113540.

[34] W. J. Drugan, J. R. Willis, A micromechanics-based nonlocal constitutive equation and estimates of representative volume element size for elastic composites, Journal of the Mechanics and Physics of Solids 44 (4) (1996) 497-524. doi:10.1016/0022-5096(96)00007-5.

[35] Z.-Y. Ren, Q.-S. Zheng, Effects of grain sizes, shapes, and distribution on minimum sizes of representative volume elements of cubic polycrystals, Mechanics of Materials 36 (12) (2004) 1217-1229. doi:10.1016/j.mechmat.2003.11.002.

[36] K. Sab, B. Nedjar, Periodization of random media and representative volume element size for linear composites, Comptes Rendus Mécanique 333 (2) (2005) 187-195. doi:10.1016/j.crme.2004.10.003.

[37] M. Ostoja-Starzewski, Material spatial randomness: From statistical to representative volume element, Probabilistic engineering mechanics 21 (2) (2006) 112-132. doi:10.1016/j.probengmech.2005.07.007.

[38] M. Ostoja-Starzewski, X. Du, Z. Khisaeva, W. Li, Comparisons of the size of the representative volume element in elastic, plastic, thermoelastic, and permeable random microstructures, International Journal for Multiscale Computational Engineering 5 (2) (2007) 73-82. doi:10.1615/IntJMultCompEng.v5.i2.10.

[39] X. Yin, W. Chen, A. To, C. McVeigh, W. K. Liu, Statistical volume element method for predicting microstructureconstitutive property relations, Computer methods in applied mechanics and engineering 197 (43-44) (2008) 35163529. doi:10.1016/j.cma.2008.01.008.

[40] C. Soize, Tensor-valued random fields for meso-scale stochastic model of anisotropic elastic microstructure and probabilistic analysis of representative volume element size, Probabilistic Engineering Mechanics 23 (2-3) (2008) 307-323. doi:10.1016/j.probengmech.2007.12.019.

[41] J. Guilleminot, C. Soize, D. Kondo, Mesoscale probabilistic models for the elasticity tensor of fiber reinforced composites: experimental identification and numerical aspects, Mechanics of Materials 41 (12) (2009) 1309-1322. doi:10.1016/j.mechmat.2009.08.004.

[42] C. Desceliers, C. Soize, Q. Grimal, M. Talmant, S. Naili, Determination of the random anisotropic elasticity layer using transient wave propagation in a fluid-solid multilayer: Model and experiments, Journal of the Acoustical 
Society of America 125 (4) (2009) 2027-2034. doi:10.1121/1.3087428.

[43] C. Desceliers, C. Soize, S. Naili, G. Haïat, Probabilistic model of the human cortical bone with mechanical alterations in ultrasonic range, Mechanical Systems and Signal Processing 32 (-) (2012) 170-177. doi:10.1016/j.ymssp.2012.03.008.

[44] M.-T. Nguyen, C. Desceliers, C. Soize, J.-M. Allain, H. Gharbi, Multiscale identification of the random elasticity field at mesoscale of a heterogeneous microstructure using multiscale experimental observations, International Journal for Multiscale Computational Engineering 13 (4). doi:10.1615/IntJMultCompEng.2015011435.

[45] M.-T. Nguyen, J.-M. Allain, H. Gharbi, C. Desceliers, C. Soize, Experimental multiscale measurements for the mechanical identification of a cortical bone by digital image correlation, Journal of the Mechanical Behavior of Biomedical Materials 63 (2016) 125-133. doi:10.1016/j.jmbbm.2016.06.011.

[46] T. Zhang, F. Pled, C. Desceliers, Robust multiscale identification of apparent elastic properties at mesoscale for random heterogeneous materials with multiscale field measurements, Materials 13 (12) (2020) 2826. doi: $10.3390 / \mathrm{ma1} 3122826$.

[47] A. Nouy, C. Soize, Random field representations for stochastic elliptic boundary value problems and statistical inverse problems, European Journal of Applied Mathematics 25 (3) (2014) 339-373. doi:10.1017/S0956792514000072.

[48] C. Soize, Uncertainty Quantification, Springer, New York, 2017. doi:10.1007/978-3-319-54339-0.

[49] M. I. Yadrenko, Spectral Theory of Random Fields, Optimization Software, 1983.

[50] Y. Rozanov, Random Fields and Stochastic Partial Differential Equations, Kluwer Academic Publishers, 1998.

[51] R. Adler, The Geometry of Random Fields, SIAM, 2010.

[52] E. Vanmarcke, Random Fields: Analysis and Synthesis, World Scientific, Singapore, 2010.

[53] M. Rosenblatt, Stationary Sequences and Random Fields, Springer Science \& Business Media, 2012.

[54] A. Malyarenko, M. Ostoja-Starzewski, Tensor-Valued Random Fields for Continuum Physics, Cambridge University Press, 2018

[55] J. Guilleminot, C. Soize, Non-gaussian random fields in multiscale mechanics of heterogeneous materials, In: Altenbach H., Ochsner A. (eds) Encyclopedia of Continuum Mechanics (2020) 1826-1834doi:10.1007/978-3662-55771-6_68

[56] C. Soize, Non gaussian positive-definite matrix-valued random fields for elliptic stochastic partial differential operators, Computer Methods in Applied Mechanics and Engineering 195 (1-3) (2006) $26-64$. doi:10.1016/j.cma.2004.12.014.

[57] J. Guilleminot, C. Soize, On the statistical dependence for the components of random elasticity tensors exhibiting material symmetry properties, Journal of Elasticity 111 (2) (2013) 109-130. doi:10.1007/s10659-012-9396-Z.

[58] J. Guilleminot, C. Soize, Stochastic model and generator for random fields with symmetry properties: application to the mesoscopic modeling of elastic random media, Multiscale Modeling \& Simulation (A SIAM Interdisciplinary Journal) 11 (3) (2013) 840-870. doi:10.1137/120898346.

[59] A. Malyarenko, M. Ostoja-Starzewski, A random field formulation of hooke's law in all elasticity classes, Journal of Elasticity 127 (2) (2017) 269-302. doi:10.1007/s10659-016-9613-2.

[60] S. C. Cowin, M. M. Mehrabadi, On the identification of material symmetry for anisotropic elastic materials, The Quarterly Journal of Mechanics and Applied Mathematics 40 (4) (1987) 451-476. doi:10.1093/qjmam/40.4.451.

[61] C. Soize, Random vectors and random fields in high dimension: parametric model-based representation, identification from data, and inverse problems, in: R. Ghanem, D. Higdon, H. Owhadi (Eds.), Handbook of Uncertainty Quantification, Vol. 2, Springer, Cham, Switzerland, 2017, Ch. 26, pp. 883-936. doi:10.1007/978-3-319-112596_30-1.

[62] C. Soize, Stochastic elliptic operators defined by non-gaussian random fields with uncertain spectrum, The American Mathematical Society Journal Theory of Probability and Mathematical Statistics. Submitted. Also in arXiv 2106.07706 [math.PR], 14 June 2021, https://arxiv.org/abs/2106.07706. (2021) 1-19.

[63] M. Bornert, T. Bretheau, P. Gilormini, Homogenization in Mechanics of Materials, ISTE Ltd and John Wiley and Sons, New York, 2008.

[64] F. Poirion, C. Soize, Numerical methods and mathematical aspects for simulation of homogeneous and non homogeneous gaussian vector fields, in: P. Krée, W. Wedig (Eds.), Probabilistic Methods in Applied Physics, SpringerVerlag, Berlin, 1995, pp. 17-53. doi:10.1007/3-540-60214-3-50.

[65] P. Krée, C. Soize, Mathematics of Random Phenomena, Reidel Pub. Co, 1986, (first published by Bordas in 1983 and also published by Springer Science \& Business Media in 2012).

[66] A. Bowman, A. Azzalini, Applied Smoothing Techniques for Data Analysis: The Kernel Approach With S-Plus Illustrations, Vol. 18, Oxford University Press, Oxford: Clarendon Press, New York, 1997. doi: $10.1007 / \mathrm{s} 001800000033$ 